\title{
Nanotechnology-based products for cancer immunotherapy
}

\author{
Forough Shams ${ }^{1} \cdot$ Ali Golchin $^{2}$ (D) Arezo Azari ${ }^{1} \cdot$ Leila Mohammadi Amirabad $^{3} \cdot$ Fateme Zarein $^{4} \cdot$ Atiyeh Khosravi $^{1}$. \\ Abdolreza Ardeshirylajimi ${ }^{5,6}$
}

Received: 17 September 2021 / Accepted: 21 October 2021 / Published online: 29 October 2021

(c) The Author(s), under exclusive licence to Springer Nature B.V. 2021

\begin{abstract}
Currently, nanoscale materials and scaffolds carrying antitumor agents to the tumor target site are practical approaches for cancer treatment. Immunotherapy is a modern approach to cancer treatment in which the body's immune system adjusts to deal with cancer cells. Immuno-engineering is a new branch of regenerative medicine-based therapies that uses engineering principles by using biological tools to stimulate the immune system. Therefore, this branch's final aim is to regulate distribution, release, and simultaneous placement of several immune factors at the tumor site, so then upgrade the current treatment methods and subsequently improve the immune system's handling. In this paper, recent research and prospects of nanotechnology-based cancer immunotherapy have been presented and discussed. Furthermore, different encouraging nanotechnology-based plans for targeting various innate and adaptive immune systems will also be discussed. Due to novel views in nanotechnology strategies, this field can address some biological obstacles, although studies are ongoing.
\end{abstract}

Ali Golchin

agolchin.vet10@yahoo.com; golchin.a@umsu.ac.ir

$\bowtie$ Arezo Azari

azari.arezo@gmail.com

1 School of Advanced Technologies in Medicine, Shahid Beheshti University of Medical Sciences, Tehran, Iran

2 Department of Clinical Biochemistry and Applied Cell Sciences, School of Medicine, Urmia University of Medical Sciences, Urmia, Iran

3 Cultured Decadence, Inc., Madison, USA

4 Department of Nanobiotechnology, Faculty of Biological Sciences, Tarbiat Modares University, Tehran, Iran

5 Urogenital Stem Cell Research Center, Shahid Beheshti University of Medical Sciences, Tehran, Iran

6 SinaCell Research and Product Center, Tehran, Iran 


\section{Graphic abstract}

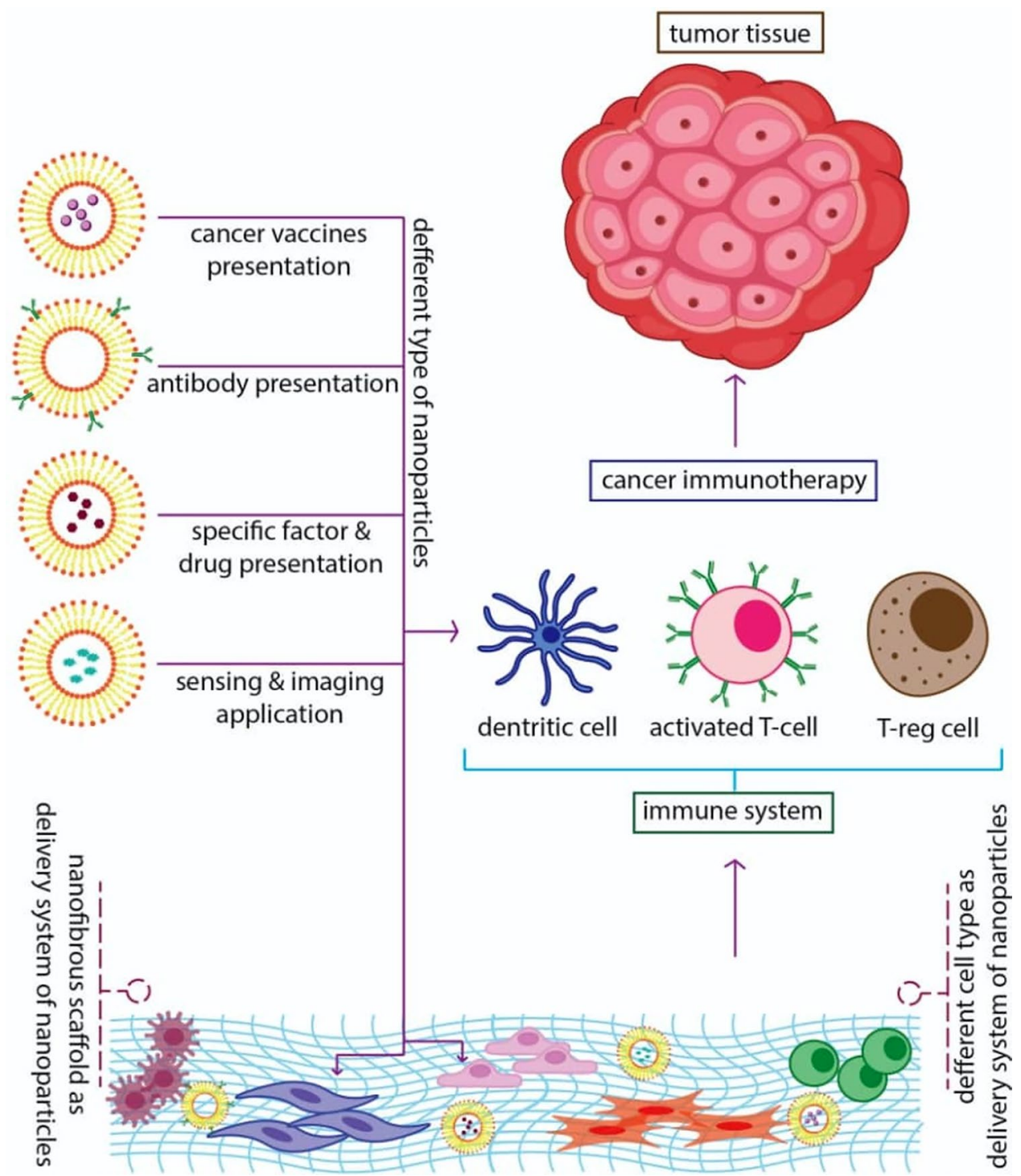

Keywords Cancer $\cdot$ Immunotherapy $\cdot$ Regenerative medicine $\cdot$ Nanotechnology $\cdot$ Neoplasms

\section{Introduction}

The host's immune system manages the health of the body and elides pathogens that threaten the general physiological condition of the host. The innate immune system has a primary role in identifying its cell-surface pattern receptors, recognizing pathogen-associated molecular patterns (PAMP) in the microorganisms and damage-associated molecular patterns (DAMP) that are released from damaged tissues to create a compatible response to microbial infections and tissue inflammations [1]. Immunotherapy, which can stimulate white blood cells to identify tumor tissues, is increasingly a promising strategy for managing cancer [2]. Immunomodulation for tumor management can be commenced as an exact tumor suppression procedure that is more effective than delivering cytotoxic agents to eradicate affected cells [3]. Its application for cancer patients is associated with long-term inhibition or even removal of tumor tissues, mainly due to the body's systematic response to such intervention and long-term memory response. Generally, immunotherapy is a therapeutic approach for cancer patients that enhances natural defenses to cope with cancer. The success of cancer immunotherapy depends on the existence of three critical agents. 
(a) Cancer antigens should be effectively performed for white blood cells, particularly antigen-presenting cells (APCs).

(b) Delivering an adjuvant to immune cells, along with antigens, shouting the immune system response.

(c) Suppression of the immune system should be regulated to achieve an anticancer immune-therapeutics response.

In this regard, nanotechnology-based products can effectively cause immune system response for each feature [4]. Recently, several cancer immunotherapies have been developed, like vaccines, chimeric antigen receptor T-cell therapy [5], immune checkpoint blockade [6], and administration of cytokines [7]. For instance, cancer vaccines are generated to strengthen cancer antigen presentation in Dendritic cells (DCs) to enhance the robustness of effector T-cell proliferation. Tumor microenvironment
(TME) modulation intends to cause the "brake" for cytotoxic T cells (CTLs) in the suppression of the immune system TME, facilitating the ability to kill their targeted cancer cells [8]. Several immunological methods have been entirely described that immunotherapies and vaccines stimulate the immune systems (both adaptive and innate) at the single-cell level. As we know, one of the most critical applications of nanotechnology-based scaffolds and nanoparticles is targeted drug delivery, which the use of this three-dimensional system for cancer treatment could create a significant revolution in cancer treatment methods (Fig. 1). In recent years, findings demonstrate that the interaction between cancer cells and immune cells is critical in cancer research to understand the immune system behaviors which can suppress cancerous growth and apply protective functions. However, recently, it is well-established known that these cancer cells are associated with
Fig. 1 Summary illustration of nanoparticles/nanofibers application for enhancing of the immune system potency against cancer

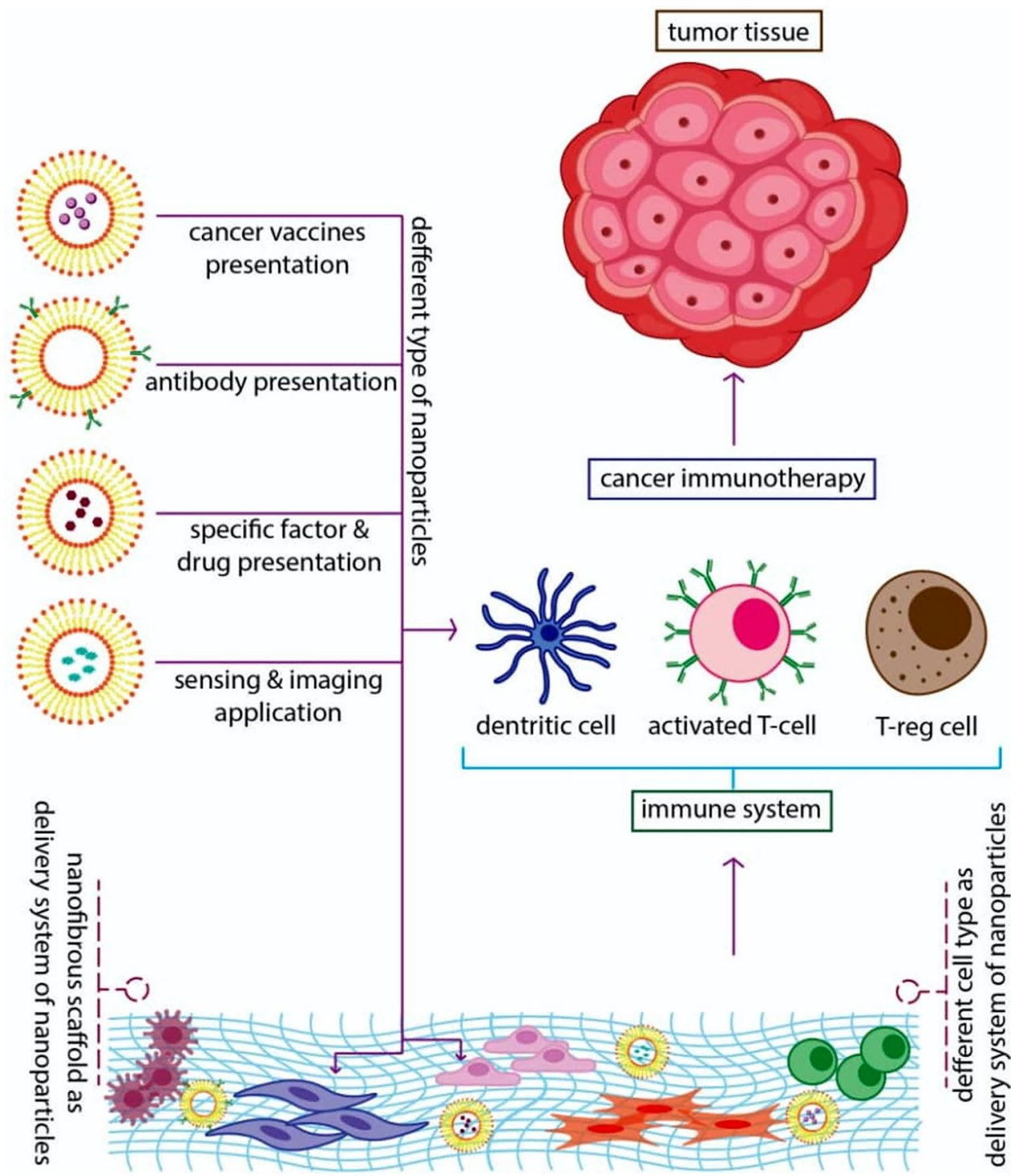


immune cells may appear acting jointly with restrict and enhances tumor development. Within the immune system, effector immune cells, including $\mathrm{CD}_{8}{ }^{+}$cytotoxic $\mathrm{T}$ cells, effector $\mathrm{CD}_{4}{ }^{+} \mathrm{T}$ cells, natural killer (NK) cells, dendritic cells, $\mathrm{M}_{1}$-polarized macrophages, and $\mathrm{N}_{1}$-polarized neutrophils are in the center of the antitumor process. In contrast, myeloid-derived suppressive cells (MDSC) or tumorassociated macrophages (TAM), their derived cytokines IL-6, tumor necrosis factor (TNF), IL-1 $\beta$, IL-23, and regulatory $\mathrm{T}$ cells (Tregs) are frequently perceived as dominant tumor-promoting immune cells $[9,10]$. Furthermore, Th17 cells, $\mathrm{CD}_{4}{ }^{+} \mathrm{CD}_{25}{ }^{+} \mathrm{Foxp}_{3}{ }^{+}$Tregs, and cytokines related to immunoregulatory like TGF- $\beta$ play dual roles concerning promoting or preventing cancer development, depending on the TME and the episodes preceding the primary promotion of tumor genesis $[9,10]$.

The cancer research field is a multidisciplinary area that contributes different scientists from diverse disciplines to the unit target. On the other hand, nanotechnology is a developing and promising field that has recently become tremendous exciting in the biomedical especially cancer therapy research. For instance, Mingxu You et al. recently developed a DNA-based gadget termed the "Nano-Claw" [11]. They combined the unique structure-switching properties of DNA aptamers with toehold-mediated strand condensation reactions to create a claw capable of performing an independent logic-based analysis of multiple cancer cell surface markers and producing a diagnostic signal and targeted photodynamic signal therapy in responding [11]. However, nanotechnology-based products (see Fig. 2) have various applications in different medical fields using various nanostructures [12]. Over the last decade, these products have been a noticeable impact in advanced medical technology, especially in multiple sectors of the cancer research fields containing the delivery mechanisms for cells, drugs, genes, and systems for protecting cells. However, structural and functional limitations in the cell range restrict the cancer therapy program's development.

Generally, there are four comprehensive strategies for using nanomaterials and nanoparticles to regulate responses of the immune system:

- The first approach contains ex vivo attaching and then internalizing immune cells, which means in vivo equipment of such cells for future injection. The nanoparticle load could deliver ingredients that stimulate the functions of immune cells or expose new functionalities to target cells.

- A next strategy contains in vivo application of "natural targeting" of nanoparticles to phagocytic cells, administration of free particles scavenged by macrophages, monocytes, neutrophils, or DCs in the blood, liver, spleen, and bone marrow, as well as other cancer cells.

- In the third method, "active" targeting is surveyed; thereby, particular antibodies or ligands on the exterior of nanoparticles are employed to achieve particular cell bindings in vivo [13].

- In the fourth strategy, the nanofibrous scaffold can be applied as a delivery tool for certain biological factors. This strategy provides a combination biologic therapy plan contain concurrent delivery of genes, cells, and other drugs $[12,14]$.

Nanotechnology-based products can exclude some functional and structural barriers in cancer immunotherapy (Fig. 3). Nanotechnology has two leading roles in vaccine structure. First, they are used as a carrier for the transfer of vaccines, and, second, due to their innate adjuvanticity, they could improve the immune responses $[15,16]$. Consequently, different nanostructures have been introduced as novel adjuvant manners for enhancing antigen delivery and presentation to APCs [13].

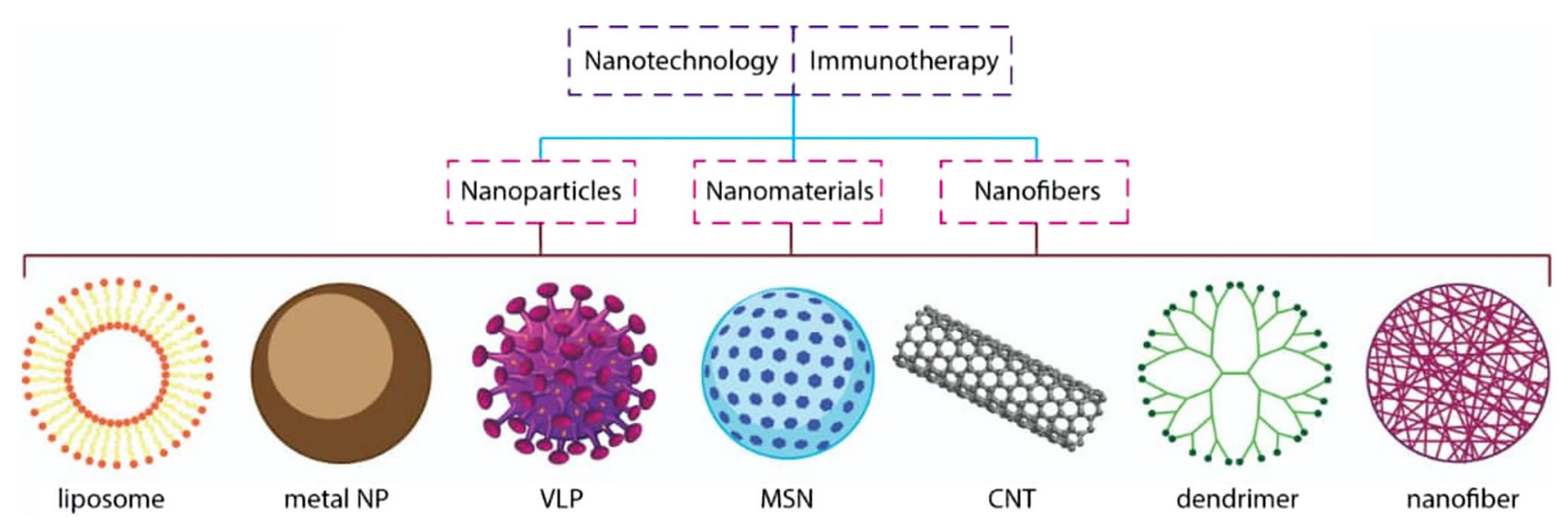

Fig. 2 Different nanomaterials with unique structures that are applied in cancer immunotherapy 


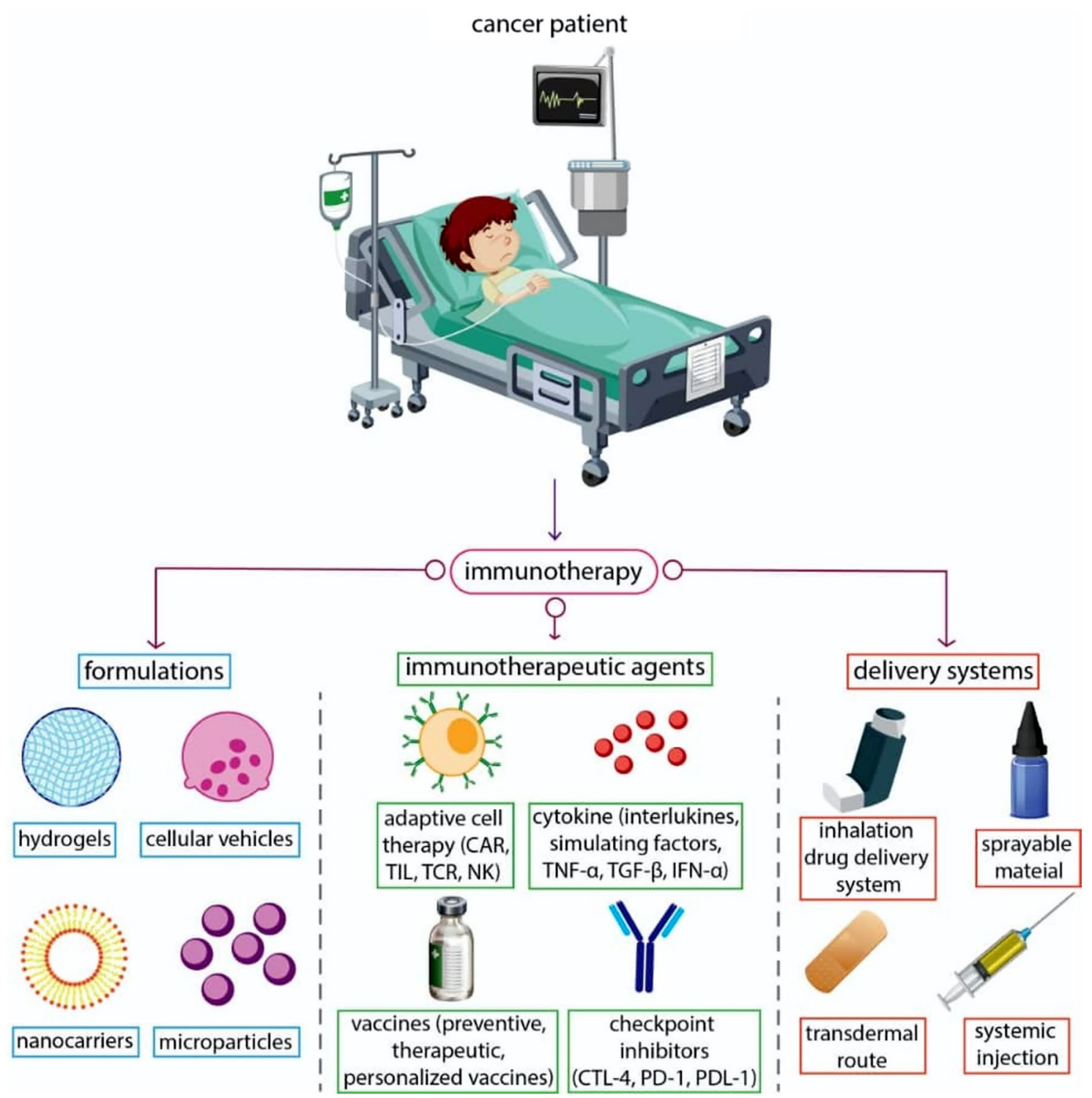

Fig. 3 Cancer immunotherapy and its multidisciplinary requirements; nanotechnology can help cancer immunotherapy in different aspects

However, some nanomaterials, due to some specific physiochemical attributes, can inherently regulate the immune response; for instance, the nanoparticle shape influences antibody and cytokine production [17]. The benefits of using nanoparticles roots in the distinctive nanoscale characteristics of conveyors, morphology, non-rigid regulation of the conveyor magnititute, and surface attributes containing targeting moieties and charge. The enhanced permeability and retention (EPR) reaction, nanoparticles due to their weak lymphatic drainage and dripping vasculature, primarily gather within tumors [18]. Moreover, nanomaterials can be modified easily, and targeting ligands already loaded on the exterior can facilitate nanomaterials take up by particular cells. Although most common immunotoxicological investigations have solely investigated the suppression of the immune system, most nanoparticle research has investigated their inflammatory characteristics.

Nonetheless, the rest of the research reported that nanoparticles are applicable for delivering drugs developed for suppressing the immune system [19]. Usage of nanomaterials to imaging agents and delivering cytotoxic agents will advantage immunotherapy. Studies demonstrate that adjuvant and antigen to APCs' concurrent delivery activates antigen-specific immune impacts. Moreover, cancer vaccines based on nanoparticles offer higher anticancer efficacy than naked curative agents shown in animal models $[13,20]$.

In the present study, the activation of the immune system in encountering cancerous conditions is investigated. After that, recent studies and prospects of nanotechnology-based cancer immunotherapy will be presented. Furthermore, 
examples of different encouraging nanotechnologies-based approaches for targeting various immune populations (either innate or adaptive) will also be discussed. We hope this paper could offer a new element of nanoparticle and nanofibrous scaffold applications in immune system function and attract readers and researchers of the cancer immunotherapy field investigating or will join this fast-developing field.

\section{Activate the immune system}

Despite many studies to understand the underlying causes of cancer, there is still no comprehensive answer to its etiology due to its complexity. Existing environmental factors in the micronucleus (micro-environment) cell, extracellular matrix, and even immune cells can influence the development and spontaneous proliferation of cancer tissues and metastatic cells and stop them. Therefore, cancerous cells and tumors should be examined in their natural niche to better study and treat cancer. The kind, density, and area of white blood cells that are called immune compositions around the tumor can predict disease progression even better than the past pathological methods; so that the immune cells around the tumor are more active than rest condition, the length of time the survival of the patient is also greater [1]. Clinical studies have determined that activating the innate antitumor immune response can treat relapsing-resistant cancers [2].

The Natural killer T cells (NKT) and $\gamma \delta \mathrm{T}$ cells have a role in immunities by interactivities with the adaptive immune cells. They are considered the connector of the adaptive and innate immune systems [1].

To suppress cancerous cells, the body employs the three strategies: (1) eliminating carcinogenic pathogens and protecting the body against the cytokines of these cells, (2) protecting the body against the pro-tumoral inflammatory environment, and (3) immunosurveillance in the activity of specific cells like $\mathrm{T}$ cells in the innate and acquired immune system that eliminates tumor cells [21].

The microenvironment of tumors usually includes an extracellular matrix and a broad spectrum of cells like fibroblasts, endothelial, immune cells (like NK, DC, polymorphonuclear leukocytes, and cytotoxic T lymphocytes [1]), cancerous tissues, and specific cytokines and receptors for these cells [22]. Accurately, type I macrophages, T helper (Th1), and NK cells are dramatically active at the cancer location to prevent its expansion [21]. Cancerous cells decrease the immune response by secreting some cytokines (called immunosuppressive challenges) [21]. If we want to explain more about the tumor microenvironment, enhanced $\mathrm{Bcl} 2$ expression increases fibroblasts and apoptosis resistance. It has also been shown that Bcl2 defends tumor cells against anticancer drugs [22]. Also, increased IL-6 expression in the aging increases TGF- $\beta$ receptors' expression, which causes tumorigenesis [22]. Many immunosuppressive factors are naturally a physiological regulatory mechanism applied by the body to prolong homeostasis to avert autoimmunity [23].

Interestingly, these are dual approaches for the system against cancer. The system has a paradoxical role, called the immunoediting hypothesis. However, in some conditions, $\mathrm{T}$ cells and antibodies against tumor-associated antigens (TAA) may induce more excellent tumor formation [1]. Thus, the immune system may enhance angiogenesis by producing and secreting some growth factors and cytokines and as well as by increasing chronic inflammation in the tumor environment, resulting in the activation of premalignant lesions [21]. In fact, chronic infections debilitate the immune system against tumor cells (an augmented cycle). For example, cancer risk is enhanced in AIDS infection (an immunosuppressive virus) that involves $\mathrm{CD}_{4+} \mathrm{T}$ cells. Thus, by secreting a large amount of interferon, the number of CD8 cytotoxic cells increases, which causes cancer tissue death cells; however, the lack of $\mathrm{CD}_{4+} \mathrm{T}$ cells prevents this reaction [21].

A variety of factors cause the host's immune system to be unable to identify cancer cells or adapt to a tumor, thereby causing the tumor to grow in the body. The presence of some parasites is one of these factors. Helminths species (a parasitic worm) can act as an immune regulator by affecting the immune system. These parasites prevent TGF- $\beta$ secretion by inhibiting the growth of $\mathrm{T}$ cells and encouraging the Treg cells growth. As a result, it obstacles the immune effect on cancer cells [21] and promotes angiogenesis [23].

The Tregs are divided into two groups: (1) natural Tregs (nTreg) that are Thymus-developed cells of $\mathrm{FoxP}_{3}$ lineage, and (2) inducible Tregs (iTreg) derived from naive $\mathrm{CD}_{4+}$ T-cell precursors under tolerogenic conditions and are upregulate $\mathrm{FoxP}_{3}$ expression [23]. Tregs regulate the immune system with 4 mechanisms: (i) Immunosuppressive molecules release, which is either soluble or membrane, (ii) straight cytolytic function, (iii) metabolic disorder, and (iv) DC suppression [23].

Another compelling factor is the age of the host. In aging, immune system activity attenuates through a decreased capacity of immunosurveillance [21, 22]. Thymopoiesis decline with an increase in age; it is thought to cause immune system damage (immunosenescence). Of course, the phenomenon of immunosenescence (refers to the gradual deterioration of the immune system brought on by biological age advancement) does not mean inactivation of all immune functions; It means that during one's life, several functions decrease, others increment, while others remain unchanged [22]. Another critical age-related factor is thymus activity. The thymus is very useful in producing $\mathrm{T}$ cells and maintaining immunity. $\mathrm{T}$ cells play an essential role as an inducer of tumor immunity [22]. This lymph node is active 
between the ages of 10 and 19 and gradually decreases with increasing age. Agents may augment the risk of cancer in aging over the 65 , such as (1) significantly reduced naive $T$ cells, (2) decreased population of lymphocyte clones, (3) an increased frequency of regulatory $\mathrm{T}$ cells that reduces the $\mathrm{T}$ cells response, (4) a low grade, proinflammatory status, and (5) increasing the number of suppressor cells that are based on myeloid, which impedes the proper functioning of T cells and produces the reactive oxygen species [24]. Increasing age causes changes in the number, phenotype, and function of leukocytes, like metabolism of glucose, PAMP signaling, phagocytic volume, and cytokine release [24]. In aging, the combination of proinflammatory cytokines [e.g. IL-1 $\beta$, IFN $\gamma$, and granulocyte-macrophage colony-stimulating factor (GM-CSF)] increases MDSCs release in the host bone marrow. It accumulates in the lymph nodes, increasing the production of MDSCs in the hose bone marrow and cumulating in the lymph. This increase in production suppresses T-cell proliferation and activity and enhances ROS production [24]. Besides, MDSCs prevent the positive effect of dendritic cell-based immunotherapies [24]. At the initial stages of tumor formation, some immune cells, like NK and $\mathrm{CD} 8+\mathrm{T}$ cells, detect them and remove them [25]. At this stage, the tumor cells can run away from white blood cells by two strategies: The first is the runaway from the immune system's identification. In this case, cancer cells prevent the identification of cytotoxic $\mathrm{T}$ cells by not expressing or reducing the expression of tumor antigens at their surface. Mutation or deletion of individual gene fragments in tumor tissues may down-regulate the presentation mechanism of the antigen, leading to resistance to T-cell effector molecules and the production of specific cytokines like IFN- $\gamma$ [25]. The down-regulated NK cells cannot identify cancer cells, especially in lung and breast cancer. In non-small cell lung cancers, only $40 \%$ of HLAs remain on the cancer cell's surface, impeding these cells' identification. The same is true for metastatic colorectal cancer, which makes resistance to T-cell transfer therapy in these patients [25]. The second is cancer cells with the secretion of immunosuppressive molecules like IL-10 and VEGF, and deterrent checkpoint molecules expressions, like PD-L1 and V domain immunoglobulin suppressor of T-cell activation, induces activity in TAMs, MDSCs, and Tregs. This activation also can induce with secreting tumor cytokines like CC-chemokine ligand 2 (CCL2), colony-stimulating factor 1 (CSF1), CCL5, CCL22, C-X-C motif chemokine 5 (CXCL5), CXCL8, and CXCL12 [26].

The tumor-associated macrophages (TAM) and tumorassociated Tregs in cancer develop an immune tolerance in the host body that destroys the effects of innate and adaptive effector immune cells [6]. Two TAMs and Tregs cells producing immunosuppressive molecules like IL-10, TGF- $\beta$ cause immune tolerance in TME (tumor microenvironment). Also, these cells can intervene in the IL-12 secretion of DCs, blocking the Th-1 response [25]. The activities of effector $T$ cells are restrained by Tregderived cytokines like IL-10, TGF-b, and IL-35 [23]. The impact of Treg on the DCs includes the following: (i) to express cytotoxic T-lymphocyte antigen 4 (CTLA-4) on Treg membrane and express CD80 and/or CD86 on DC membrane causes cell-cell signaling, to increase the production of 2,3-dioxygenase (IDO) in DCs, (ii) to decrees DC capacity to activate effector $\mathrm{T}$ cells by inhibiting costimulatory molecules, (iii) to suppress of DC maturation via IL-10/TGF-b signaling, (iv) Treg-DC interactions mediated by lymphocyte-activation gene 3(LAG3) [23] In one study shown in EMT6 breast cancer, inhibition of TGF- $\beta$ secretion increases response to treatment with antiPD-L1, leading to improved patient survival [25].

In breast cancer, hypoxia and stimulating angiogenesis can cause Treg to migrate to the tumor site. Whereas hypoxia augments CCL28 expression at the cancer cell surface, this ligand interacts with the CCR10 receptor on the Treg surface (with the cd4+/CD25+/Foxp3+marker). Also, in ovarian cancer, CCL28 expression is related to hypoxia-inducible factor 1a (HIF-1) expression, indicating a poor prognosis of the disease [23].

DCs and macrophages are APCs. In general, DCs are considered APCs with the highest effectiveness, required in activating CTLs through MHC class I molecules and costimulator (like CD80 and CD86) and in initiating an adaptive immune response. Aging may decrease the antigen-presenting capacity of APCs and the existence of co-stimulatory pathways on their exterior. Much experimental evidence suggests that one of the causes of decreased APC activity in aging is decreased proteasome expression. APCs with TLRs can detect self and non-self-pathogen-associated structures. The expression level of these receptors decreases with aging. Moreover, cytokine production essential for both distinction and functions of APCs, like IL-4 and IL-12, reduces in elders [25].

To sum up this section, cancer tissues can escape from the immune system in various ways. As a result, most safe immune processes, especially those embedded in complex and solid tumors, are only effective for a small number of patients. Therefore, the provision of immune cells to address the obstacles created by the suppression of the tumor's immune cells is a pivotal immunotherapy approach. Empirical evidence has shown that immune-stimulating molecules that are delivered using nanoparticles and scaffolds with greater precision and intensity can stimulate the immune system to deliver these molecules through the solution; thus, its antitumor effects are also higher. Hence biologists and tissue engineers are working to determine which cells, pathways, and tools can enhance the effectiveness of this stimulation and activation of the immune system. 


\section{Nanoparticle and nanofibrous scaffold preparation}

According to the recent advances in nanotechnology and its application in medicine and daily life, the last century can be named nanotechnology. There are several nanoparticles and nanofibrous scaffold fabrication methods such as emulsification and dispersion polymerization, solvent evaporation and solvent extraction processes, phase separation, interfacial polymer deposition following solvent displacement, spray drying, and also several industrial methods. Moreover, there are several nanofibrous mats fabrication methods such as Electrospinning, Selfassembly, Cell-matrix adhesion, Drawing and Patterning, which can produce by natural or synthetic polymers and the composite of them in the presence and absence of the immune-cells encapsulated nanoparticles. Due to specific cellular uptake and tissue bio-distribution, nanoparticles could have wide application in tissue and cell-based therapy related to cancer immune therapy $[12,27]$. Several new studies have introduced nanoparticle applications as organ/cell-specific carriers and detection, imaging, tissue engineering, bio-fabrication, and therapeutic purposes [12, $28,29]$. In the following, we focus on studies and ideas that present nanotechnology-based products for developing cancer immunotherapy.

\section{Nanotechnology and cancer immunotherapy}

\section{Nanoparticles and cancer immune-therapy}

Nanoparticles are synthetic particles made up of polymers, lipids, or metals and are nanoscale in size. These nanoparticles can be used in biodegradable carriers and therapeutic agents that cause high-dose drug release in the target area (Fig. 1). The surface-to-volume ratio of these nanoparticles has enabled these materials to be coated with various ligands, including antibodies and aptamers, thereby facilitating the interaction with the desired molecules, including receptors located on the exterior of target cells. Moreover,

\section{Nano-Engineered MSCs as Active Targeting Drug Delivery Vehicles}
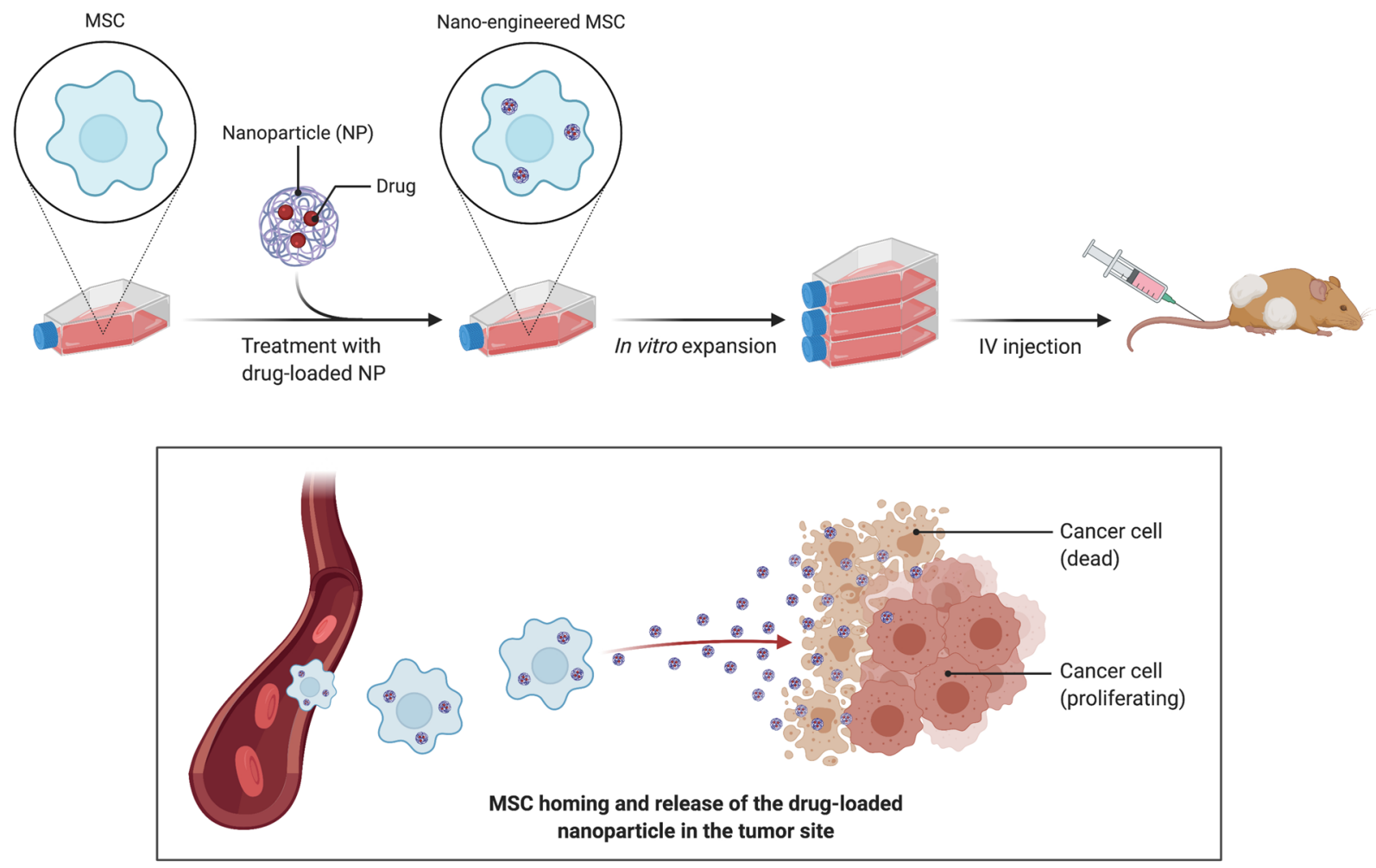

Fig. 4 Combining nanotechnology and cell therapy to generate "nano-engineered" mesenchymal stem cells. This biological product would be able to actively target the tumor site and protect the drug- loaded nanoparticle from vascular filtration and macrophage clearance; The figure is made with biorender (https://biorender.com/) 
nanoparticles can be applied in effective engineering cells as active targeting drug delivery vehicles (Fig. 4). Cell-based therapies, particularly stem cells, have led to optimism in treating different diseases, especially cancer [30-32]. This advantage of nanoparticles also can be valuable in engineering different cell-based therapy products for developing cancer immunotherapy.

Furthermore, nanoparticles can enhance the pharmacokinetics of maximum drug loading [3], which effectively increases their ability to target cancer cells that escape the immune system. Labeling nanoparticles for specific receptors on cancer cells reinforces cellular absorption by aggregating the desired molecule and releasing it in place. In contrast, leukocytes can actively move through these chemokine gradients onto these nanoparticles towards tumor cells and consequently stimulate these cells as ultimate targets. Therapeutic delivery systems are one of the main challenges in therapeutic approaches $[12,33]$. The delivery of immunestimulatory drugs to antitumor immune cells brings about cancerous cells rather than delivering cytotoxic drugs, which results in almost no risk of recurrence.

Consequently, the successful delivery of immune-stimulating molecules to a small number of immune cells can significantly enhance antitumor and therapeutic efficacy while administering a large number of cytotoxic drugs and delivering them to many tumor cells may not change even in the progression of cancer. It is due to the cancerous cells being heterozygous and maybe a drug for several types of deadly clones, but not for many fatal ones, and/or some cloning cells survive due to lack of medication. In this regard, even if there is a resistant clone or insufficient penetration of the drug, few cancer cells survive, the risk of relapse may be $100 \%$ [34, 35]. Conversely, immune cells with memory cells can provide a more consistent and lasting response to all other types of therapies. Targeting nanoparticles for dendritic cells are more likely than targeting these nanoparticles for cancer cells $[15,36]$. This can be attributed to the activities of our immune system. The secondary lymphoid organs, especially the spleen that is full of dendritic cells, accumulate in these structures due to the stomatal structure. Moreover, secondary lymphoid organs do not have physical inhibition due to a dense extracellular matrix for immune cell entry in solid tumor tissues $[15,36]$.

Dendritic cells are the primary triggers for acquired immune responses and are, therefore, the most important targets for anticancer nano-drugs. Simultaneous systemic administration of antigen and adjuvants can result in antigens delivery to some DCs and delivery of adjuvant to other dendritic cells. Delivery of antigen to DCs in the absence of adjuvant results in immunologic tolerance and prevents the strengthening of antitumor responses. Simultaneous placement of antigens and adjuvants in a particle can simultaneously deliver these two components of the same dendritic cell, resulting in enhanced stimulation of specific antigens of $\mathrm{CD} 8^{+} \mathrm{T}$ cells, a primary antitumor immunity mediator. Research has shown that the efficient release of the antigen from a particle (as an antigen source) to dendritic immune cells by enhancing the antigen delivery to immune cells can lead to further increase of the killer T cells and improve cancer, including Papilloma [37, 38].

On the other hand, a series of dendritic cells are essential in the induction, stimulation and immune activities regulation. Plasmacytoid dendritic cells can be converted from inactive to the active and immune-stimulating state by attracting Toll-like receptor agonists (TLRs). Hence, using several dendritic cell subgroups, the effectiveness of antitumor drugs can be maximized by simultaneously stimulating hemorrhagic and cellular immune responses [39]. Zhang et al. have recently reported that their nanoparticle formulations can effectively improve the severe toxicities of immune-stimulatory agents for antitumor immunity. They designed nanoparticle (PEGylated) liposomes bearing surface-conjugated interleukin 2 (IL-2) and anti-CD137, and observed that anchoring IL-2 and anti-CD137 on the surface of nanoparticles allows such immune agonists to aggregate in cancer tissues by systemic IV injection quickly. This study introduces a general approach for using nanoparticles to apply the potent inducing function of immune agonists in cancer immunotherapy [40]. Therefore, nanoparticles provide an interactional condition for the cooperation of two or multiple chemotherapeutic factors. In another study, polyethylene glycol-modified poly (lactic-co-glycolic acid) nanoparticles were used to increase the synergic effect of IRAK1/4 inhibitor and ABT-737 in T cell acute lymphoblastic leukemia (T-ALL) mice. They were reported to enhance the survival duration of T-ALL on the mouse model [40].

\section{Nanostructural scaffolds and cancer immune-therapy}

In addition to nanoparticles, scaffolds with a nano structure can also be used to produce anticancer vaccines. The selfassembling (SA) is a considerable change by which targets of nanophase/molecules evolve to instant categories [41]. Different SA systems are introduced, ranging from copolymers in blocks to 3D cell culture scaffolds. These structures can manage various materials, such as different polymers, and use them in cancer therapy and tissue engineering [41]. Nanostructural scaffolds are applied to address some of the clinical obstacles to immunotherapies that use $\mathrm{T}$ cells and result in a new perspective on this evolving area at the interface of cancer immunotherapy and nanotechnology-based products. Moreover, scaffolds could be usefully similar to conventional dendritic cell-based vaccines, which require the isolation, ex vivo manipulation and re-entry of dendritic cells into the patient's body for the use of them. 
Polymeric and hydrogel scaffolds that containing inflammatory cytokines, tumor antigens, and topical immune-compromised signals in one place can be transplanted or injected as an appropriate topical medication. A study has shown that hydrogel scaffolds containing manipulated mesenchymal stem cells secreting antitumor proteins showed a robust antitumor effect on the tumor site [39]. Another study was shown that insertion of lysed tumor particles, $\mathrm{CpG}$ oligonucleotide, GM-CSF chemokine, TLR9 agonist in a porous PLGA scaffold and its subcutaneous transplantation could activate and homing of the dendritic cells. Consequently, it triggered the destruction of tumors in the place or the farthest position [42]. This ability, because of delivering antigen and adjuvant messenger to an immune cell simultaneously [42]. As previously mentioned, this simultaneous delivery of the vaccine components as a solution into a single cell with very low probability occurs.

Solid tumors generally create a dense environment around them and suppress the immune system at the tumor site and prevent activating ACT, which escapes the immune system via this way. The transfer of lymphocytes to biodegradable polymeric scaffolds can promote the growth and release of tumor-degrading T-cells in the tumor and most efficiently reduce the risk of cancer recurring rather than when $T$ cells are used systemically or even topically applied [43, 44].

Hence nanoparticles and nanostructural scaffolds as valuable tools can be applied in cancer Immune-therapy. In the following, we review the nanotechnology-based product's interaction with immune system agents in the cancerous condition during its immunotherapy strategies.

\section{Nanomaterials for modulating innate immune system}

Nanoparticles can increase the immunomodulator's delivery to immune cells and recuperate the effect of immunotherapy. Most studies indicate that it is feasible to apply immunotherapy by adjusting innate immune cells. Nanoparticles can modulate various innate immune cells, including monocytes, NK cells, TAMs, neutrophils, DCs, and MDSCs [45]. For instance, TAMs can act as antigen-presenting cells and produce different solubles to interact with other immune cells. In addition, they play a crucial role in cancer immunotherapy [45]. For instance, Song et al. [46] used the extra benefits of the high accumulation of TAMs in hypoxic regions of cancer tissues and elevated reactivity of manganese dioxide nanoparticles $\left(\mathrm{MnO}_{2} \mathrm{NPs}\right)$. They intended to produce hydrogen peroxide $\left(\mathrm{H}_{2} \mathrm{O}_{2}\right)$ for the concurrent modulation of $\mathrm{pH}$ and production of $\mathrm{O}_{2}$ for effective reduction of tumor hypoxia by targeting the delivery of $\mathrm{MnO}_{2} \mathrm{NPs}$ to the hypoxic area. Hence, they reported that combined management of cancer tissues with Man-HA- $\mathrm{MnO}_{2}$ NPs and Doxorubicin considerably increases apparent diffusion coefficient (ADC) values of breast cancer cells.

Moreover, cancer cell oxygenation was increased, hypoxia-inducible factor- $1 \alpha$ (HIF- $1 \alpha)$ down-regulated, and cancer cell proliferation and growth were inhibited compared to the sole chemotherapy [46]. Silva et al. [47], in a study on the utilization of dextran crosslinked-iron oxide nanoparticles in Lewis lung carcinoma tumor-bearing mice, reported changed phenotypes of TAM. In the nanoparticletreated group, the variation of TAM to proinflammatory type was significant. Thus, this research requires consideration in that inorganic iron oxide nanoparticles operated as both an image contrast agent and a TAM-reprogramming agent [47].

As well as, Liu et al. showed that self-crosslinked redoxresponsive nanoparticles based on galactose-functionalized n-butylamine-poly (1-lysine) -b-poly (l-cysteine) polypeptides (GLC) coated with DCA-grafted sheddable poly(ethylene glycol) PEG-PLL (sPEG) copolymers was successfully used to deliver miR155 to TAMs [48]. Recently it was reported that miR155 engagaed in reducing cytokine secretion and to polarize M2-type macrophages toward M1 type. Management of miR155-loaded sPEG/GLC (sPEG/ GLC/155) nano-complexes enhanced miR155 expression in TAMs about 100-400 folds both in vivo and in vitro. The sPEG/GLC/155 also effectively repolarized immunosuppressive TAMs to antitumor M1 macrophages through increasing M1 macrophage markers (IL-12, iNOS, MHC II) and suppressing $\mathrm{M} 2$ macrophage markers (Msr2 and $\mathrm{Arg} 1$ ) in TAMs. It was found that treatment with sPEG/GLC/155 increased activated NK cells and T lymphocytes in tumors, which resulted in strong reversion of the tumor [48].

In comparison to TAMs, few research described modulating NK cells, which can produce proteins like proteases and perforin to marginal cancer tissues and enhance the permeability of the membrane of target cells [49]. In this regard, a series of nanomaterials are investigated for their properties to modulate or stimulate the NK cells [50]. Jang et al. studied nanoparticles with magnetic properties to regulate the transfer of NK cells to tumor tissues [50]. They separated and loaded NK cells with nanoparticles with magnetic properties using a magnetic field. They intravenously injected the human B cell lymphoma-bearing mice to expose them to an external magnetic field. Simultaneously by exposure magnetic field, a robust fluorescent color signal was identified in the cancer tissues. Their findings proved the migration of NK cells. This procedure lets us open another clinical therapeutic intervention with the lessened toxicity of the nanoparticles and increased infiltration into target cells [50].

MDSCs are another component of innate immunity that cannot be differentiated into mature forms, such as granulocytes and dendritic cells. It is found that the accommodation of MDSCs at tumor cells can include active NK cells and suppress $\mathrm{T}$ cell proliferation while enhancing the regulatory 
$\mathrm{T}$ cells distinction. The nanomaterial-mediated selective modulation of MDSCs at tumor tissues was associated with improved immune system suppression and showed a novel method for immunotherapy. Again, evidence regarding the nanomaterials to modulate MDSCs are not sufficient [51]. Since MDSCs cooperate in the negative regulation of antitumor immune reactions, decreasing MDSCs are a significant point to invert suppression of the immune system in the tumor microenvironment. Kong et al. in a study described a type of biodegradable hollow mesoporous silica nanoparticle (dHMSN) for co-arranged delivery of all-trans retinoic acid (ATRA), doxorubicin (DOX), and interleukin-2 (IL-2) to get increased antitumor efficiency in chemo-immunotherapy [52]. It was found that ATRA was helpful for differentiating the MDSCs from antitumor immune cells like mature dendritic cells (DCs), granulocytes, and macrophages and ameliorates the tumor-specific immune response. Hence, ATRA has a remarkable ability to regulate MDSC-induced immunosuppression [53]. According to interventions that are based on MDSC modulation, particular MDSC-targeting strategies require further evaluations. For instance, Liu et al. applied MDSC aptamer modified liposomes. Based on their findings, T1 aptamer was successfully binded to both MDSCs and tumor cells [54]. Besides, studies in the differentiation field [52] found that the lipid nanomaterials were employed for modulating MDSCs also could deliver cytotoxic anticancer pharmaceutical ingredients. The delivery of cytotoxic anticancer drug-loaded nanoparticles may increase the ability to distinct MDSCs and kill them. Future research on differentiation of MDSCs can use MDSC-specific nanomaterials, which may utilize favorable activities without destroying MDSCs [52, 54].

Neutrophils are essential for innate immune responses and advocacy against annoying infections. In response to chemotaxis, they can quickly transmigrate to infected or injured areas. In this regard, the accumulation of nanocarriers containing circulating neutrophils can provide a chance for therapeutic drug delivery management. Hence, the application of neutrophil-mediated drug delivery is investigated for cancer immunotherapy [55]. In another research by Chu et al., they used a strategy (i.e. monoclonal antibody TA99 specific for gp75 antigen of melanoma) for snatching neutrophils in vivo by nanoparticles to transfer therapeutics into the tumor [56]. In a mouse model of melanoma after injection, the photosensitizer (pyropheophorbide-a-loaded albumin nanoparticles) was anticipated to adjoin to neutrophils in the blood. To manage the neutrophils to cancer cells, the TA99, as co-injected, can modulate neutrophils to melanoma cancer cells by affecting antibody-dependent cell-mediated cytotoxicity. Hence, this study determines a novel way to cure cancer through NP hitchhiking of the immune system to increase pharmaceutical ingredients transfer to cancer cells [56]. As well, Chu et al. conducted another study about the remodeling of tumor microenvironments that can enhance the delivery of nanoparticles [57]. Since the CD11b antibody was applied as a signal for activating neutrophils, they used this antibody with gold nanoparticles for increasing the neutrophils infiltration into cancer cells. A drug delivery platform consisted of NPs coated with anti-CD11b antibodies that target activated neutrophils. To increase neutrophils infiltration to cancer infected tissues, pyropheophorbide was administered to mice and clarified at $660 \mathrm{~nm}$. The investigators assumed that, in tumor cells, photosensitization-induced might perform the gold nanoparticle-bound neutrophils to permeate the tissues. In conclusion, the group treated by CD11b-antibody-decorated gold nanoparticles demonstrated prolonged survival and reduced tumor growth those that received pegylated gold nanoparticles [57].

In all peripheral tissues, DCs are innate-similar cells, which are interfacing cells that associate the innate and adaptive immune reactions because they can activate native $\mathrm{T}$-cells and B-cells (sometimes). These cells were gathering antigens from the circumambient fluid and remaining on stable vigilance for "danger signals"-molecular motifs implicating pathogen invasion or tissue damage. As a response caused by the innate immune system, DCs are capable of expressing various diagnostic receptors, like a stimulator of interferon genes (STING), TLRs, a retinoic acid-inducible gene I (RIG-I)-like receptors (RLRs), NODlike receptors (NLRs), and C-type lectins. These molecules are responsible for activating DCs in cellular damage or pathogen exposure [45]. Several immunomodulators like STING agonists and TLR agonists are created to increase the DCs' activities within cancer management. Regrettably, such immunomodulators have side effects. In addition, they are unstable. For solving this significant challenge, investigators are looking to use nanomaterials for delivering these immunomodulators. Hence, nanomaterials are intended to co-deliver immunomodulators for maximizing the effect of immunomodulators [58].

Nanoparticles increase DC activation effects on APCs by encapsulating or representing danger signals. Also, by "programming" the stimulation of DCs, nanoparticle formulations can directly affect humoral induction and cellular immunity to immunotherapies and vaccines [13]. For instance, Luo et al. reported in the study that activation of STING promotes the antigen-presenting capability and maturation of DCs [59]. In this study, a synthetic polymeric nanoparticle, PC7A NP, was produced with a peptide antigen as a nanovaccine that causes a potent cytotoxic T-cell activity, with a limited systemic cytokine expression. Upon administration, these nanoparticles were spread in the nodes of the lymph. According to the findings, PC7A presented a high binding property for STING. Thus, according to in vitro investigation based on DCs derived from bone marrow, these nanoparticles presented an adjuvant impact on DC evolution 
and increased maturation markers' expression, comprising CD80 and CD86 [59]. Furthermore, in mouse models of colorectal cancer, melanoma, and lymphoma, vaccination with PC7A nanoparticles was more significant in tumor prevention than injecting vaccines that contained free antigen [13].

In another study, TLR7/8 agonist, imiquimod, treating basal skin carcinoma was approved by the FDA, and it is sold as a topical cream under the name Aldara ${ }^{\circledR}[60]$. Imiquimod was co-encapsulated with the photothermalresponsive factor that means indocyanine green in the hydrophobic portion of biodegradable poly (lactic-co-glycolic) acid nanoparticles. Intravenous injection of indocyanine green/imiquimod-loaded polymeric nanoparticles demonstrated accumulation in tumor tissues. Afterward, according to the findings, irradiation could separate the primary cancer tissues of CT26 colon tumor-bearing mice. Indeed, this removal resulted in increased antigen presentation and DCs' maturation [60]. Hence, available evidence has centralized on the innate immune cell biology mechanisms and their connection with cancer (Table 1).

\section{Nanomaterials for modulating adaptive immune system (AIS)}

T-cells and B-cells are components of the AIS. These cells express various clonal antigen receptors and identify an extensive group of antigens expressed by either cancer cells or other pathogens. Indeed, these cells can evolve to memory cells, which can 'remember' prior exposures with antigens and begin a quick immune reaction against those that are formerly faced [13].

T-cells have a significant role in the ability of the body to remove tumors and intracellular pathogens. Treatment with adoptive T-cell transfer or checkpoint blockade antibodies are primarily dependent on the strength of the cytotoxic CD8 + T-cells spoil cancer cells and infiltrate tumors, showing significant responses in the clinic. Also, abnormal T-cell reactions cause severe autoimmune disorders like MS and Type 1 diabetes. Hence, some strategies are needed for modulating the activities of T-cells, either increasing or preventing their role in a variety of diseases [82]. Tumorantigen-specific T-cells that naturally increase in patients, expanded by a cancer vaccine, or presented by adoptive cell treatment may eradicate cancer tissues. The critical point is applying strategies for developing these T-cells, particularly in in vivo or preventing signals that indicate the suppression of the immune system. Hence, nanoparticle delivery factors have been investigated to increase the activity of T-cellbased immunity in various mechanisms.

In this regard, Stephan et al. manipulated cytokine encapsulating multilamellar lipid nanoparticles and linked them (chemically) to T-cells ex vivo previous to adoptive transfer [83]. They reported that conjugating nanoparticles to free thiols on T-cell surface proteins caused minimum particle internalization after several days of culture. Nanocarriers, meanwhile, produce encapsulated cytokines to stimulate modulated autocrine of cell exteriors' receptors. Since T-cells contained nanoparticle "backpacks" carrying stimulatory cytokines, this procedure could show 80 -fold enhanced T-cell proliferation in vivo and notable developments in the ACT efficacy, with no harmful effect (i.e. toxicity). Thus, particle-decorated T-cells could capture tumor tissues. Besides, it was shown that cell-bound nanoparticles could be moved from the cell's surface into the tumor cell/T-cell interface (i.e. synapse-directed drug delivery) [83]. Kwong et al. have also used nanoparticles to carry protective or stimulatory cues to T-cells in the tumor microenvironment [84]. They created immunostimulatory liposomes while the surface of PEGylated liposomes was conjugated with IL-2 and anti-CD137. Such particles are intended to administer high doses of IL-2 and anti-CD137 into tumors without systemic diffusion. Therefore, local stimulation of T-cell was done without systemic toxicity. After intratumoral administration of anti-CD137 and IL-2 fixed on liposomes into melanomas, which caused ameliorated proportions of tumor-infiltrating $\mathrm{CD}_{8}{ }^{+} \mathrm{T}$-cells to regulatory T-cells (T-regs), treated about $70 \%$ of subjects, and primed T-cells that were not close to the administration site to stop the proliferation of other tumor cells [84].

Nanoparticles are also used for treating autoimmune diseases by enabling preferred re-modification of autoreactive T-cells. For example, T1D is because of $\mathrm{CD}_{8}{ }^{+} \mathrm{T}$-cells, which identify several epitopes secreted by islet cells of the pancreas. Therefore, Tsai et al. reported that activation of self-antigen-specific $\mathrm{CD}_{8}{ }^{+} \mathrm{T}$-cells through iron oxide nanoparticles linked with pMHC-NPs increased the number of autoregulatory memory-like T-cells [85]. Hence, such autoregulatory memories-like T-cells inhibited the stimulation of autoreactive $\mathrm{CD} 8^{+}$T-cells via knocking out of APCs capable of presenting auto-antigens. Table 2 shows other nanomaterials used for regulating adaptive immune (T-cells) cells for immunotherapy [85].

The humoral immune response (B cells) plays a unique role by exploiting both antigen-specific effector cells and APCs. B cells maintain the extracellular spaces of the host and are critical for free pathogens clearance. Prevention (i.e. through vaccines) is the best approach to get prolonged conservation against contagious agents using humoral immune reactions. Licensed vaccines were protected via stimulating neutralizing antibody reactions. Therefore, introducing nanoparticles to increase the activation and engagement of antigen-specific B-cells plays a vital role in vaccine development [108]. Nanoparticles of biological sources like viruslike particles (VLPs), exosomes, inactivated and weakened viruses have been widely applied in various vaccines to 
Table 1 Nanomaterials used as a goal of modulating innate immune cells for immunotherapy

\begin{tabular}{|c|c|c|c|c|c|}
\hline Nanomaterial & Targeted cell & Purpose & Tumor models & Cargo (Payload) & References \\
\hline Liposome & TAM & TAM reduction & KPC & Clodronate & {$[61]$} \\
\hline Carboxylated polystyrene & TAM & TAM activation & E0771/E2 & & {$[62]$} \\
\hline Glucomannan polysaccharide & TAM & TAM reduction & S180 & Alendronate & {$[63]$} \\
\hline $\begin{array}{l}\text { Hyaluronic acid-protamine @ } \\
\text { cationic liposome }\end{array}$ & TAM & TAM activation & B16F10 & CD47 siRNA & {$[64]$} \\
\hline $\begin{array}{l}\text { Poly(b-amino ester) } \\
\text { g-poly(ethylene glycol)-g- } \\
\text { histamine }\end{array}$ & TAM & TAM reprogramming & B16F10 & IL-12 & {$[65]$} \\
\hline $\begin{array}{l}\text { Poly }(\text { L-lysine }) \text { b-poly }(\mathrm{L}- \\
\text { cysteine) @ Poly(ethylene } \\
\text { glycol)-poly(Llysine)-g- } \\
\text { (1,2dicarboxyliccyclohexene) }\end{array}$ & TAM & TAM reprogramming & B16F10 & $\operatorname{miR} 155$ & [48] \\
\hline $\begin{array}{l}\text { M2-like TAM dual-targeting } \\
\text { nanoparticles (M2NPs) }\end{array}$ & TAM & TAM reduction & B16F10 & $\begin{array}{l}\text { Anti-CSF-1R siRNA } \\
\text { (siCD115) }\end{array}$ & {$[66]$} \\
\hline $\begin{array}{l}\text { Iron oxide/carboxymethyldex- } \\
\text { tran nanoparticle }\end{array}$ & TAM & TAM reprogramming & KP1 & ferumoxytol & {$[67]$} \\
\hline $\begin{array}{l}\text { Hyaluronic acid }-\mathrm{MnO}_{2} \text { nano- } \\
\text { particle }\end{array}$ & TAM & TAM reprogramming & $4 \mathrm{~T} 1$ & & [46] \\
\hline $\begin{array}{l}\text { cyclodextrin nanoparticles } \\
\text { (CDNPs) }\end{array}$ & TAM & TAM reduction & RAW 264.7 and B16.F10 & TLR7/8-agonist & {$[68]$} \\
\hline $\begin{array}{l}\text { Lipid NPs (C12-200, choles- } \\
\text { terol, PEG-DMG) }\end{array}$ & TAM & TAM reduction & EL4, CT26 & CCR2 siRNA & [69] \\
\hline Liposome/sialic acid & TAM & TAM eradication & S180 sarcoma & Epirubicin & {$[70]$} \\
\hline $\begin{array}{l}\text { High-density lipoprotein-like } \\
\text { NPs (HDL) }\end{array}$ & MDSCs & MDSC reduction & B16F10 & $\begin{array}{l}\text { lipophilic fluorophore } \\
\text { dialkylcarbocyanine } \\
\text { (DiD) }\end{array}$ & [71] \\
\hline $\begin{array}{l}\text { Micelles of pply propylene } \\
\text { sulfide (PPS) }\end{array}$ & MDSCs & MDSC depletion & B16F10, E.G7-OVA & 6-thioguanine(TG) & {$[72]$} \\
\hline Liposome/DSPE-PEG-PDP & MDSCs & MDSC differentiation & $4 \mathrm{~T} 1$ & Complement C3 & [73] \\
\hline Mesoporous Silica @ liposome & MDSCs & MDSC differentiation & $\mathrm{B} 16 \mathrm{~F} 10$ & All trans retinoic acid & {$[52]$} \\
\hline Liposome & MDSCs & MDSC reduction & EG07-OVA B16F10 & Lauroyl gemcitabine & {$[74]$} \\
\hline Liposome & MDSCs & MDSC depletion & $4 \mathrm{~T} 1$ & Doxorubicin & {$[54]$} \\
\hline $\begin{array}{l}\text { UCNP-PEG-PEI (UPP) nano- } \\
\text { particles } \\
\text { upconversion }(U C N P) \\
\text { polyethylene glycol }(P E G) \\
\text { polyethylenimine }(P E I)\end{array}$ & DC & DC activation & $\mathrm{C} 57 \mathrm{BL} / 6$ & Antigen ovalbumin (OVA) & [75] \\
\hline Poly(lactic-co-glycolic) acid & DC & DC activation & СТ26 & $\begin{array}{l}\text { Indocyanine green, imiqui- } \\
\text { mod }\end{array}$ & [76] \\
\hline HDL mimicking nanodisc & DC & DC activation & B16F10 & CpG ODN, antigen peptide & {$[20]$} \\
\hline MoS2 PEG nanosheets & DC & DC activation & C57BL/6 & $\mathrm{CpG}$ & [77] \\
\hline $\begin{array}{l}\text { A polymer-templated protein } \\
\text { nano-ball with thhemaggluti- } \\
\text { nin1 }(\mathrm{H} 1)(\mathrm{H} 1-\mathrm{NB})\end{array}$ & DC & DC Stimulation & $\mathrm{C} 57 \mathrm{BL} / 6$ & Ovalbumin (OVA) & [78] \\
\hline $\begin{array}{l}\text { Poly(ethylene glycol)-b-poly-2- } \\
\text { (hexamethylene-imino) ethyl } \\
\text { methacrylate }\end{array}$ & DC & DC activation & B16F10 MC38 TC & Antigen peptide & [59] \\
\hline $\begin{array}{l}\text { D- } \alpha \text {-tocopherol polyethylene } \\
\text { glycol succinate (TPGS) }\end{array}$ & DC & DC activation & CT26-FL3 & $\begin{array}{l}\text { The micellar delivery of } \\
\text { quercetin }(\mathrm{Q}) \text { and alanto- } \\
\text { lactone }(\mathrm{A}) \\
(\mathrm{QA-M})\end{array}$ & [79] \\
\hline $\begin{array}{l}\text { Binary cooperative prodrug } \\
\text { nanoparticle (BCPN) }\end{array}$ & DC & DC activation & $4 \mathrm{~T} 1$ & $\begin{array}{l}\text { (oxaliplatin)OXA and } \\
\text { NLG919 }\end{array}$ & {$[80]$} \\
\hline $\mathrm{Fe}_{3} \mathrm{O}_{4} @ \mathrm{SiO}_{2}$ nanoparticle & NK cell & NK cell migration & RPMI8226 & & {$[50]$} \\
\hline Cationic liposome & NK cell & NK cell activation & CMT167 & Plasmid encoding TUSC2 & [49] \\
\hline
\end{tabular}


Table 1 (continued)

\begin{tabular}{|c|c|c|c|c|c|}
\hline Nanomaterial & Targeted cell & Purpose & Tumor models & Cargo (Payload) & References \\
\hline Cationic liposome & Neutrophil & $\begin{array}{l}\text { Neutrophil mediated } \\
\text { delivery }\end{array}$ & G422 & Paclitaxel & [81] \\
\hline Denatured albumin + TA99 Ab & Neutrophil & $\begin{array}{l}\text { Neutrophil mediated } \\
\text { delivery }\end{array}$ & B16 & Pyropheophorbide-a & {$[56]$} \\
\hline Gold nanoparticle & Neutrophil & $\begin{array}{l}\text { Neutrophil mediated } \\
\text { delivery }\end{array}$ & $3 \mathrm{LL}$ & & [57] \\
\hline
\end{tabular}

ameliorate the antibody activities. Such vaccines' proficiency relies on repetitive particulate antigens for activating responses of the B-cell, which act simultaneously with the monovalent ligation of the B-cell receptors (BCR) [109].

A little evidence is available regarding the tumur stimulated immunologic functions of B cells, which consist of a different subset of functions, and their modulation has an essential effect on tumoricidal function. Moreover, those stimulated by the BCR pathway, TLR, and microRNA pathway can show antitumor immunity by secreting antibodies, chemokines, and cytokines, which are naive APCs, and organizing tertiary lymphoid structures [110].

Plasma cells that are a subset of B cells can generate antibodies that cause antitumor reactions like complementdependent cytotoxicity (CDC) and antibody-dependent cell-mediated cytotoxicity (ADCC). Furthermore, mature follicular B cells can evolve to Be-1 and -2 and generate cytokines like IL-2, -12 , TNF- $\alpha$, IFN- $\gamma$ for increasing antitumor Immunity of NK and T cells. By stimulating the CD40CD40 ligand (CD40L) signaling pathway, B cells turn into naive APCs in cancer tissues. They can conserve the survival and growth of $\mathrm{T}$ cells that can infiltrate cancer cells for lasting antitumor reactions.

Antitumor immunity, which is negatively regulated by regulatory B cells (B regs), is triggered by a variety of mechanisms:

(1) B regs secret mediators that can suppress the immune system like cytokines and IDO-1, which prevent the activation and proliferation of $\mathrm{NK}$ as well as T cells.

(2) B regs inactivate cells that can stimulate the immune system by expressing regulators of the immune system like PD-1.

(3) B regs upgrade the progression of cancer tissues by producing TGF- $\beta$ for EMT.

(4) When B regs produce the death-stimulating molecule Fas ligand (FASL), they, in turn, stimulate the death of effector T cells.

As specified above, using antibodies against programmed cell death-1 (PD-1), programmed cell death ligand-1 (PDL1), and the role of PD-1 inhibitors in non-small cell lung cancer, melanoma, and bladder, renal, and head and neck cancers, as well as Hodgkin's lymphoma, is well proved. Recently, various immunotherapeutic antibodies accompanied with nanoparticles as drug delivery were confirmed by the US FDA (Table 3). Thus, approaches applied to transform or target B regs can create a therapeutic potential for improving antitumor immunotherapy. For example, Schmid et al. targeted $\mathrm{CD} 8^{+}$T-lymphocytes by the PD- 1 antibody after eliminating the $\mathrm{Fc}$ fragments and connecting to poly (D, L-lactic-co-glycolic acid) (PLGA) nanoparticles [111]. Furthermore, the nanoparticle covers an inhibitor of TGF- $\beta$, which is useful for immunosuppressive tumor microenvironment preservation and immune adjuvants TLR7/8 agonists. Then, PD-1 positive T-lymphocytes were targeted in tumor tissue and blood. The TGF- $\beta$ inhibitors and TLR7/8 agonists increased the immune reactions, enhanced the vulnerability of the tumor to subsequent anti-PD-1 therapies, and enhanced the ratio of CD8 T-lymphocytes that can infiltrate the tumor. They reported a macrophage dual-targeted CpGloading nanoparticle created by mannosylated carboxymethyl Chitosan and hyaluronan (HA). This nanosystem caused enhanced secretion of proinflammatory cytokines, phosphoinositide 3-kinase/Akt signal pathways, activated NF- $\mathrm{KB}$, and stimulated Fas/FasL-mediated cell death in models developed for breast cancer [111]. As mentioned above, $\mathrm{B}$ cells are indirectly affected by this nanosystem like that some of these immunity mechanisms that regulate negatively via regulatory B cells. When B-lymphocyte receptors are interconnected, B-cell growth and differentiation into plasma cells that can produce particular antibodies are begun. Temchura et al. [112] were demonstrated biodegradable calcium phosphate nanoparticles that have protein antigens on their exterior and provided the efficacy of the B-cell stimulation following the presentation to the nanoparticles mentioned above. The nanoparticles were activated with the model antigen Hen Egg Lysozyme (HEL) and preferentially internalized and limited by the tumor antigen-specific B-lymphocytes. Co-generation of HEL-specific B-cells with activated nanoparticles enhanced exterior production of B-cell stimulator markers and induced high humoral immunity. Nanoparticles can cross-link B-cell receptors effectively at the exterior of antigen-matched B-cells. Furthermore, it is found that their efficiency is significantly higher (about 100-times) in activating B-cells, compared to the soluble 


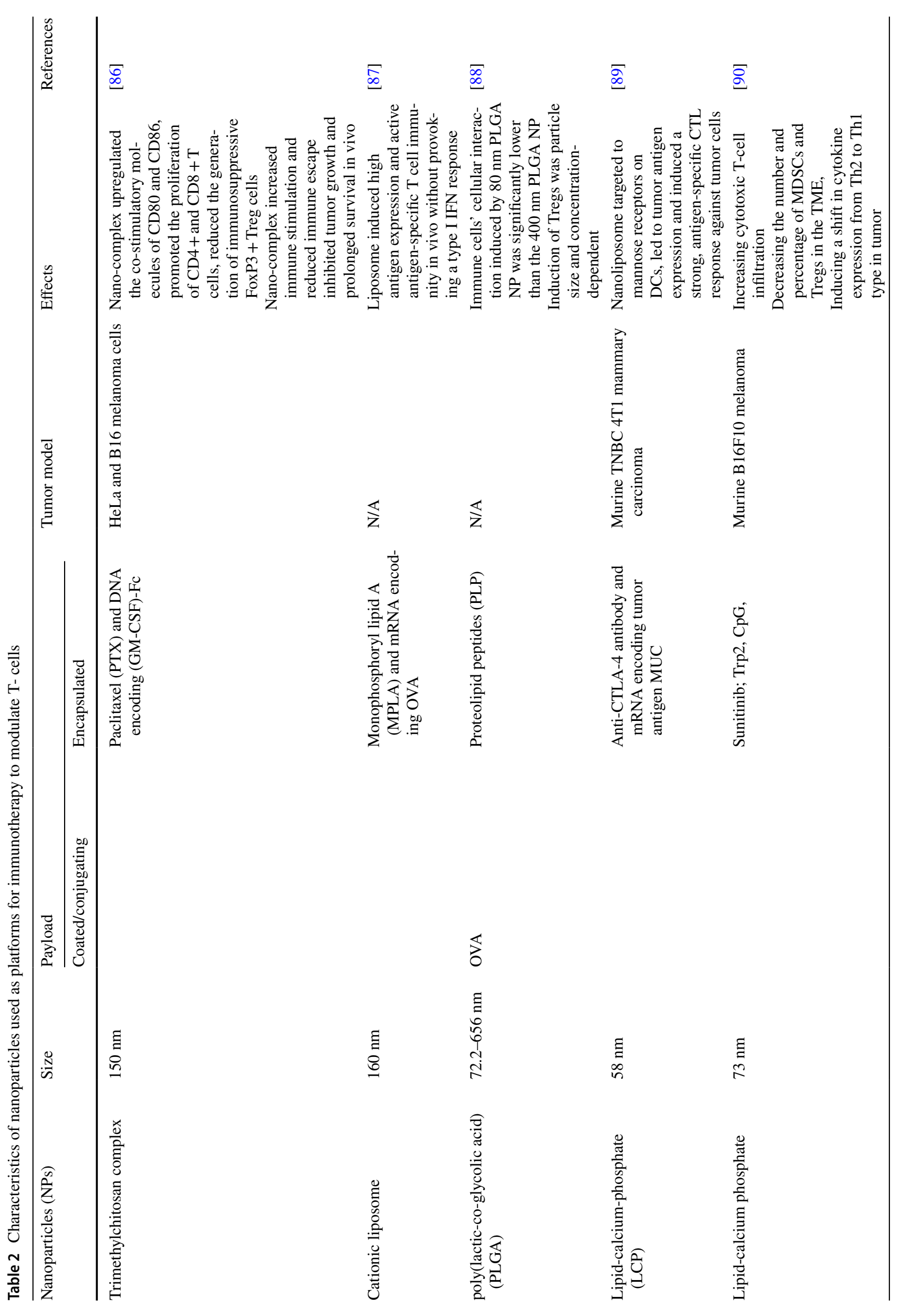




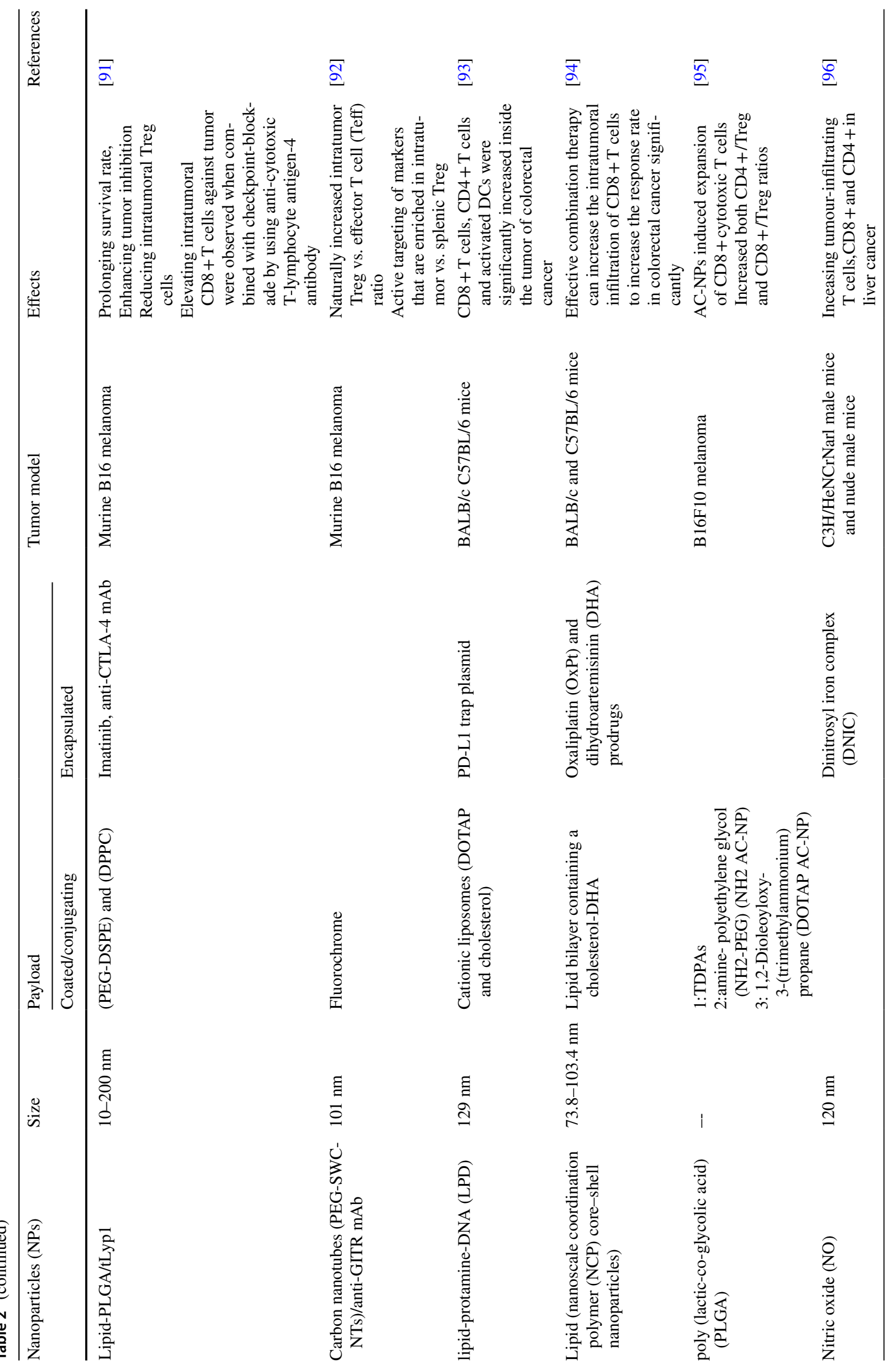




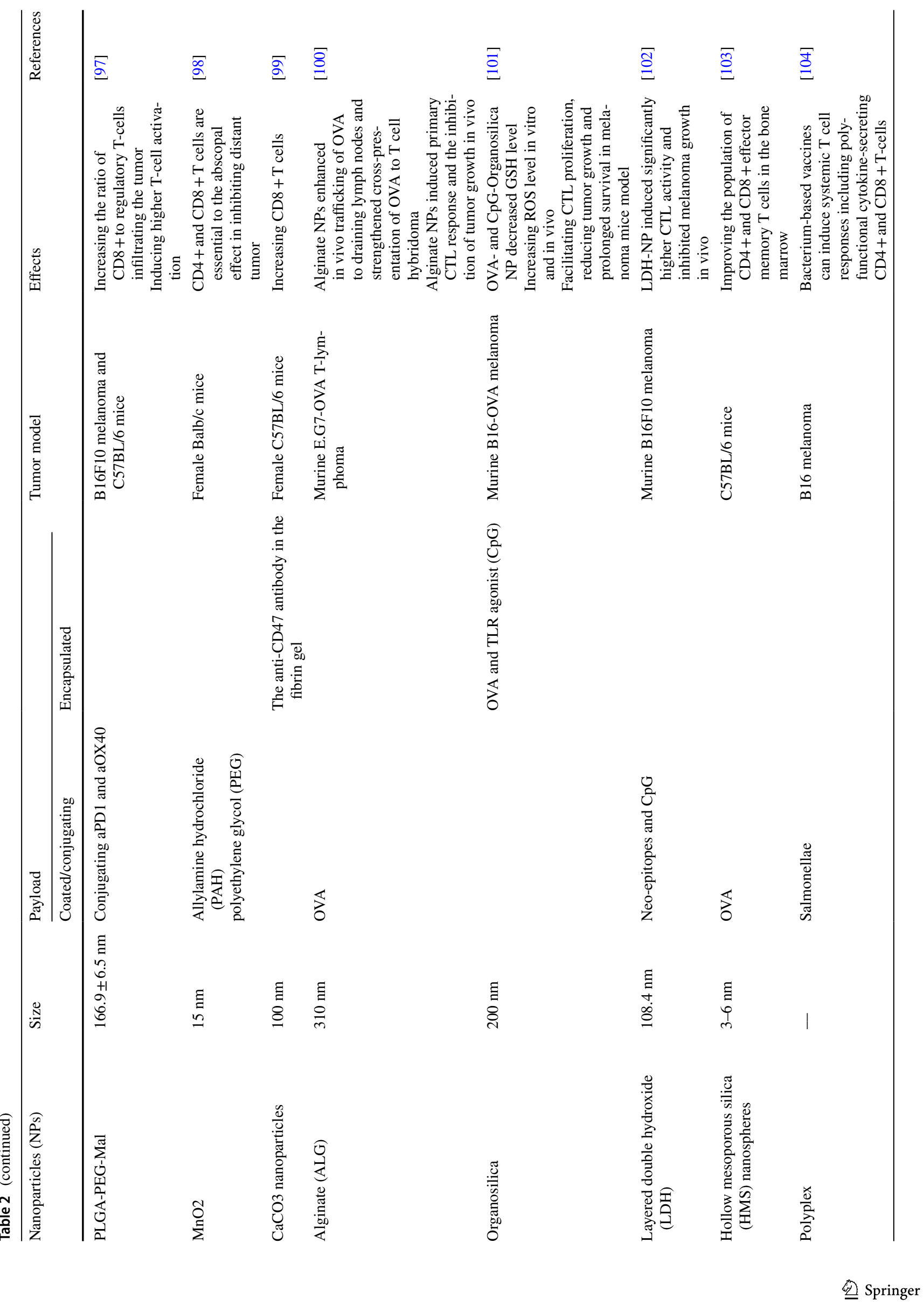




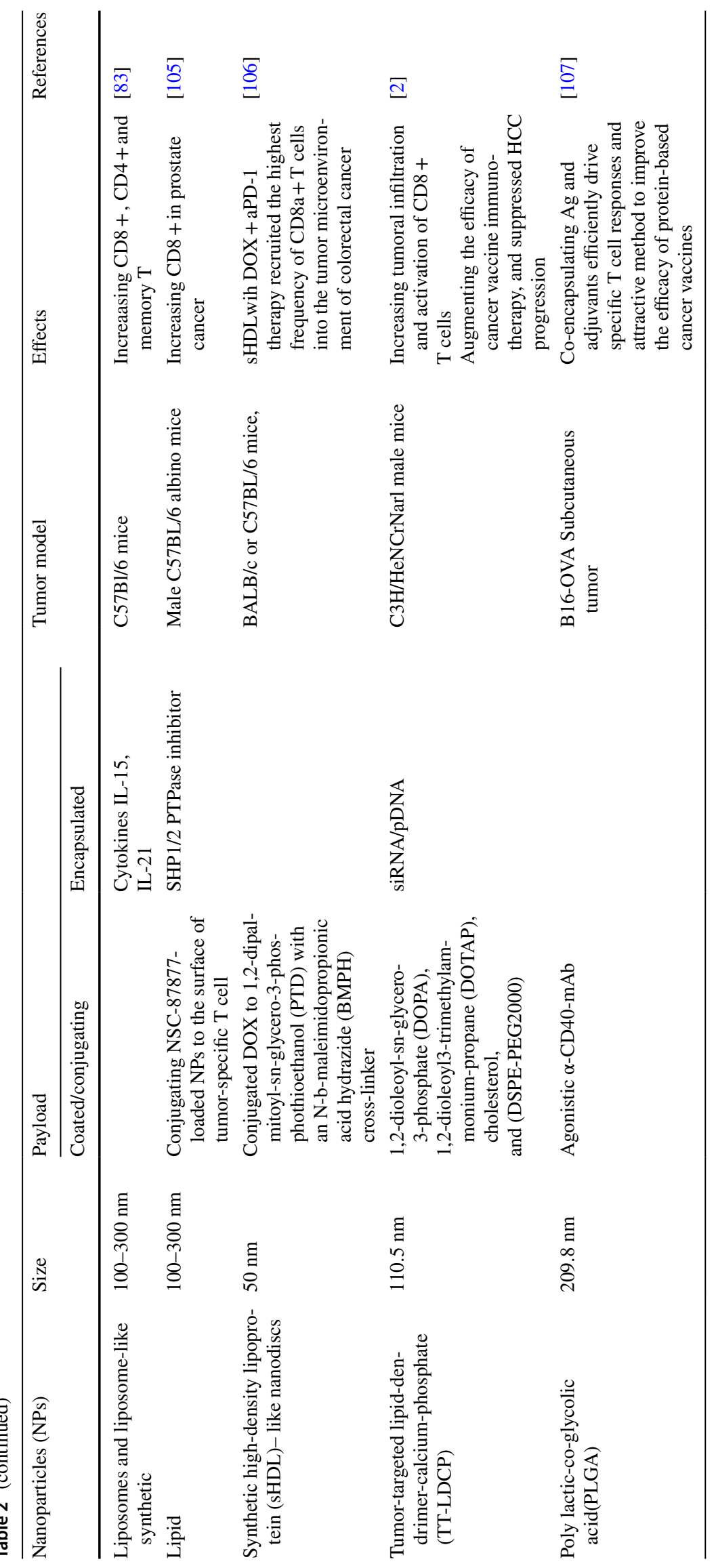


Table 3 List of cancer immunotherapeutic antibodies along with nanoparticles as drug delivery approved by the US Food and Drug Administration (FDA)

\begin{tabular}{|c|c|c|c|c|}
\hline Drug/treatment & Type of cancer & Drug type (target) & Approval & Year \\
\hline Durvalumab (Imfinzi®) & Locally advanced or metastatic bladder cancer & Monoclonal antibody (PD-L1) & Approved & 2017 \\
\hline Avelumab (Bavencioß) & Locally advanced or metastatic bladder cancer & Monoclonal antibody (PD-L1) & Approved & 2017 \\
\hline Nivolumab (OPDIVO®) & Classical Hodgkin lymphoma (cHL) & IgG4 kappa monoclonal antibody (PD-1) & Approved & 2016 \\
\hline Atezolizumab (Tecentriq®) & Locally advanced or metastatic bladder cancer & Monoclonal antibody (PD-L1) & Approved & 2016 \\
\hline Pembrolizumab (Keytruda $\left.{ }^{\circledR}\right)$ & $\begin{array}{l}\text { Advanced refractory melanoma and non-small } \\
\text { cell lung cancer }\end{array}$ & Monoclonal antibody (PD-L1) & Approved & 2014 \\
\hline Nivolumab (Opdivo®) & $\begin{array}{l}\text { Unresectable or metastatic melanoma squa- } \\
\text { mous non-small cell lung cancer }\end{array}$ & Monoclonal antibody (PD-L1) & Approved & 2014 \\
\hline
\end{tabular}

shape of tumor antigen. Hence, these nanoparticles covered with protein antigens are appropriate vaccines for humoral immunity [112].

As efficient adjuvants in the formulation of antitumor vaccine are critical, Yan et al. were examined two-layered double hydroxide (LDH) nanoparticles and nanosheets (NSs) as adjuvants to stimulate the immune reactions for antitumor purposes [113]. They showed that immunogen ovalbumin (OVA) carried by both nanomaterials could induce more robust humoral and cell-mediated immune reactions, combined with an immune stimulant (i.e. TLR9 ligand $\mathrm{CpG}$ ), as documented using increased levels of IgG1, IgG2a, and interferon- $\gamma$. Also, LDH NSs presented enhanced function to increase particular antibody activities compared with LDH NPs. It was found that immunizing mice with OVA-CpG vaccines developed with both nanomaterials, presented more robust inhibition of the inoculated tumor proliferation. Besides, an increased survival is reported [113]. In another study, Martucci et al. demonstrated a novel drug delivery mechanism for active targeting of B-cell lymphoma [114]. A novel customized B-cell lymphoma treatment, which uses a site-specific receptor-mediated drug delivery system and natural silica-based nanoparticles (diatomite), have been altered to target the antiapoptotic factor B-cell lymphoma/leukemia 2 (Bcl2) with small interfering RNA (siRNA). Aggressive murine A20 lymphoma cells, using as ligand an idiotypespecific peptide (Id-peptide) endowed with high affinity into the B-cell receptor. This Id-peptide was applied as a homing to assure the specific targeting of target cells. Diatomite nanoparticles (DNPs) loaded with siRNA directed against $\mathrm{Bcl} 2$ and conjugated with an Id-peptide, powerfully produced in the majority of B-cell lymphomas, are provided as a ligand-mediated delivery system. Hence, specific uptake of nanoparticle guided using the Id-peptide was increased by three times in target cells than nonspecific myeloma cells. When a random control peptide was exploited, instead of Id-peptide, and siRNA was administered to modified nanoparticles, the specific internalization yield was increased fourfold [114].
In conclusion, gene silencing has a high biological role and provides a chance for customized lymphomas. As mentation above, B cells are closely related to APCs and T cells, and most of their functions in cancer suppression are based on modulating and regulating $\mathrm{T}$ cells. So, $\mathrm{B}$ cells are frequently considered as helper cells during immunotherapy.

\section{B cell-targeting nanoparticles for immunotherapy in infectious diseases}

Another aspect of immunotherapy using nanoparticles can be observed in the immune response to infectious diseases. The immune reaction to local microbial agents and vaccines has induced the investigation of synthetic nanoparticles that contain identical structural features in antigen represent to enhance humoral immunity. Engineered nanoparticles offer flexibility over the manner of particulate antigen presentation. On the other hand, the surface presentation of peptide and protein antigens on nanoparticle exterior imitating bacterial and viral agents can conjugate and stimulate antigen-specific B cells with an increased rate compared to encapsulated antigens [115].

Friede et al. showed that, at similar doses, liposomes with surface-bound peptide antigens exploited a more robust B-cell reaction than those with encapsulated peptide antigens [116]. Protein conjugated on the exterior of calcium phosphate nanoparticles increases linking of BCR in vitro, with increased effectiveness in stimulating antigen-specific B cells than soluble proteins. Furthermore, many physicochemical factors can affect the function of nanoparticles to involve B-cells. In this regard, Stefanick et al. demonstrated how peptide linker length, peptide capacity, PEG coating density, peptide hydrophilicity, and PEG linker length could considerably change cellular absorption for peptide-functionalized liposomes that bind to targeted cells [117].

More silica, gold, and calcium phosphate nanoparticles $(\mathrm{CaP})$ have been applied for developing vaccines in the past decade. Compared to biologic or nanoparticles based on polymer, they pose more benefits in feasibility, generation, 
safety, and storehouse costs. For instance, Zilker et al. reported that $\mathrm{CaP}$ coated with proteins effectively activates antigen-specific B-cells in vitro [118]. They showed that immunization with $\mathrm{CaP}$-nanoparticles elicits more robust responses compared to monovalent antigens. Furthermore, the function of the CaP-nanoparticles with TRL-ligands can modulate the level of mucosal IgA antibodies and the IgG subtype response. Also, the functionalization of $\mathrm{CaP}$ with $\mathrm{CpG}$ or T-helper cell epitopes provides overcoming the absence of T-helper cells. Hence, their results indicate that $\mathrm{CaP}$ nanoparticle-based $\mathrm{B}$-cell targeting vaccines functionalized with TLR-ligands can be used as a multiplatform to dramatically modulate and induce humoral immune responses in vivo [118].

Another study was reported by Kasturi et al., who developed a vaccine based on nanoparticles, like a virus, in composition and size [119]. They used a biodegradable synthetic PLGA to synthesize $~ 300 \mathrm{~nm}$ nanoparticles with TLR4, TLR7, or both ligands with an antigen. Immunization of mice with this composition considerably enhanced antigen-specific serum IgG titer against influenza virus hemagglutinin than immunization with a single TLR agonist. A remarkable characteristic of the immune response stimulated by this vaccine is the induction of long-lived germinal centers (GCs) and continuous antigen-specific B and $\mathrm{T}$ cell responses, similar to those observed with viral infections. In conclusion, encapsulated TLR4 and TLR7 ligand in PLGA formulations increase the GC mechanism of memory B cell development and prolonged plasma cell reactions [119]. Almost all studies have found that nanoparticle surface-indicated antigens can increase high-affinity antibodies compared to soluble antigen immunization and the formation of the germinal center (GCs).

\section{Conclusion and promising perspective of future}

In sum, nano-based cancer immunotherapy is still in its initial stage but has succeeded in improving cancer vaccines' safety and efficacy. This fast-developing field has attracted many researchers to make applicable the theoretical thesis in the experimental exploitation. Nevertheless, there are 295 clinical trials recorded on the NIH clinical trials website (https://clinicaltrials.gov/) that aim to assess the impact of diverse nanoparticles on various cancers ("Search of: Nanoparticles|Cancer-Search was done at Date 2021March 11th).

Nanotechnology combined with biocomputing capabilities has the potential to enable the development of sophisticated nanorobotic devices for a wide range of biomedical applications, including intelligent sensors and new therapeutic agents. DNA/RNA-based computing techniques have been developed recently that may have innovative clinical applications, such as analysing cells and delivering agents [120]. However, the effectiveness and complexity of this field remain largely unknown in cancer immunotherapy. Cellular "hitchhiking," or the use of cells as vehicles for the co-delivery of cancer therapeutics and nanoparticles to the target tissue, is a novel strategy for extending the circulation of nanoparticles [121].

Perhaps it can be concluded that significant primary progress in the administration of nanoparticles in immunotherapy for those with cancer includes illustrating and adjusting the function of immune cells in the context of the tumor microenvironment. However, polymeric nanoparticles are the most investigated nanomaterials in cancer immunotherapy for delivery systems of drugs and immune factors. Moreover, the importance of gold nanoclusters, liposomes, inorganic porous material in this field should not be overlooked [122]. The biomaterial research field's development currently provides significant opportunities for successful Nanobiotechnology's clinical trials as an advanced technology in medicine. According to our review, over the last 20 years, many nanoparticle-based ingredients, including encapsulated or conjugated cytotoxic drug delivery systems, have entered clinical trials. There is great promise for nanotechnology to achieve clinical translations of biomedical applications and clinically relevant diagnostic and therapeutic multifunctional systems. Due to the unique features of these products, some critical issues are entirely investigated. The new investigation focused on producing nontoxic, non-immunogenic, stable, completely biocompatible, and reusable administration with little or no side effects for cancer patients. These features make nanotechnology-based products an excellent candidate for personalized medical planning. Indeed, some of the effective nanoparticle therapeutic outcomes used in cancer immunotherapy help quickly translate engineered nano-immunotherapeutics into cancer management clinics.

Acknowledgements The authors did not recipe any financial support for the research. I want to acknowledge and give my thanks to Elham Geravandi, who help us for revising the manuscript.

Author contributions AG contributed to the study's conception and design. The first draft of the manuscript was written by AG, LMA and AA, and all authors commented on previous versions of the manuscript. Finally, all authors read, approved, and revised the final manuscript.

\section{Data availability NA.}

\section{Declarations}

Conflict of interest The authors declare no potential conflicts of interest.

Informed consent Not applicable. 
Research involving human and/or animals participants The authors declare that there is no research on human participants or animals.

\section{References}

1. Liu Y, Zeng G (2012) Cancer and innate immune system interactions: translational potentials for cancer immunotherapy. J Immunother 35:299-308

2. Huang K-W, Hsu F-F, Qiu JT et al (2020) Highly efficient and tumor-selective nanoparticles for dual-targeted immunogene therapy against cancer. Sci Adv 6:eaax5032. https://doi.org/10. 1126/sciadv.aax5032

3. Goldberg MS (2015) Immunoengineering: how nanotechnology can enhance cancer immunotherapy. Cell 161:201-204. https:// doi.org/10.1016/j.cell.2015.03.037

4. Park W, Heo YJ, Han DK (2018) New opportunities for nanoparticles in cancer immunotherapy. Biomater Res 22:24. https:// doi.org/10.1186/s40824-018-0133-y

5. Singh AK, McGuirk JP (2020) CAR T cells: continuation in a revolution of immunotherapy. Lancet Oncol 21:e168-e178

6. Kalbasi A, Ribas A (2020) Tumour-intrinsic resistance to immune checkpoint blockade. Nat Rev Immunol 20:25-39

7. Chulpanova DS, Kitaeva KV, Green AR et al (2020) Molecular aspects and future perspectives of cytokine-based anti-cancer immunotherapy. Front Cell Dev Biol 8:402

8. Melero I, Gaudernack G, Gerritsen W et al (2014) Therapeutic vaccines for cancer: an overview of clinical trials. Nat Rev Clin Oncol 11:509-524. https://doi.org/10.1038/nrclinonc.2014.111

9. Zamarron BF, Chen W (2011) Dual roles of immune cells and their factors in cancer development and progression. Int J Biol Sci 7:651-658. https://doi.org/10.7150/ijbs.7.651

10. Lei X, Lei Y, Li JK et al (2020) Immune cells within the tumor microenvironment: Biological functions and roles in cancer immunotherapy. Cancer Lett 470:126-133

11. You M, Peng L, Shao N et al (2014) DNA "Nano-Claw": logicbased autonomous cancer targeting and therapy. J Am Chem Soc 136:1256-1259. https://doi.org/10.1021/JA4114903

12. Golchin A, Hosseinzadeh S, Roshangar L (2017) The role of nanomaterials in cell delivery systems. Med Mol Morphol 51:112. https://doi.org/10.1007/s00795-017-0173-8

13. Irvine DJ, Hanson MC, Rakhra K, Tokatlian T (2015) Synthetic nanoparticles for vaccines and immunotherapy. Chem Rev 115:11109-11146. https://doi.org/10.1021/acs.chemrev.5b00109

14. Ardeshirylajimi A, Golchin A, Khojasteh A, Bandehpour M (2018) Increased osteogenic differentiation potential of MSCs cultured on nanofibrous structure through activation of Wnt $/ \beta$ catenin signalling by inorganic polyphosphate. Artif Cells Nanomed Biotechnol 46:S943-S949. https://doi.org/10.1080/ 21691401.2018.1521816

15. Yan S, Zhao P, Yu T, Gu N (2019) Current applications and future prospects of nanotechnology in cancer immunotherapy. Cancer Biol Med 16:486-497

16. Qian H, Liu B, Jiang X (2018) Application of nanomaterials in cancer immunotherapy. Mater Today Chem 7:53-64

17. Smith MJ, Brown JM, Zamboni WC, Walker NJ (2014) From immunotoxicity to nanotherapy: the effects of nanomaterials on the immune system. Toxicol Sci 138:249-255. https://doi.org/ 10.1093/toxsci/kfu005

18. Maeda H, Tsukigawa K, Fang J (2016) A retrospective 30 years after discovery of the enhanced permeability and retention effect of solid tumors: next-generation chemotherapeutics and photodynamic therapy-problems, solutions, and prospects. Microcirculation 23:173-182. https://doi.org/10.1111/micc.12228
19. Azzi J, Tang L, Moore R et al (2010) Polylactide-cyclosporin A nanoparticles for targeted immunosuppression. Faseb j 24:39273938. https://doi.org/10.1096/fj.10-154690

20. Kuai R, Ochyl LJ, Bahjat KS et al (2017) Designer vaccine nanodiscs for personalized cancer immunotherapy. Nat Mater 16:489-496. https://doi.org/10.1038/nmat4822

21. Jacqueline $\mathrm{C}$, Bourfia $\mathrm{Y}, \mathrm{Hbid} \mathrm{H}$ et al (2016) Interactions between immune challenges and cancer cells proliferation: timing does matter! Evol Med Public Heal 2016:299-311. https://doi.org/10. 1093/emph/eow025

22. Malaguarnera L, Cristaldi E, Malaguarnera M (2010) The role of immunity in elderly cancer. Crit Rev Oncol Hematol 74:40-60

23. Facciabene A, Motz GT, Coukos G (2012) T-regulatory cells: key players in tumor immune escape and angiogenesis. Cancer Res 72:2162-2171

24. Bowdish DME (2013) Myeloid-derived suppressor cells, age and cancer. Oncoimmunology. https://doi.org/10.4161/onci.24754

25. Gonzalez H, Hagerling C, Werb Z (2018) Roles of the immune system in cancer: From tumor initiation to metastatic progression. Genes Dev 32:1267-1284

26. Mariathasan S, Turley SJ, Nickles D et al (2018) TGF $\beta$ attenuates tumour response to PD-L1 blockade by contributing to exclusion of T cells. Nature 554:544-548. https://doi.org/10.1038/natur e25501

27. Golchin A, Hosseinzadeh S, Jouybar A et al (2020) Wound healing improvement by curcumin-loaded electrospun nanofibers and BFP-MSCs as a bioactive dressing. Polym Adv Technol 31:1519-1531. https://doi.org/10.1002/pat.4881

28. Zhan Y, Cao X, Cao X et al (2017) Silica cross-linked micellar core-shell nanoparticles encapsulating IR-780 with strong bright and good biocompatibility for optical imaging in vivo. J Biomed Nanotechnol 13:144-154. https://doi.org/10.1166/jbn.2017.2332

29. Escudero-Francos MA, Cepas V, González-Menéndez P et al (2017) Cellular uptake and tissue biodistribution of functionalized gold nanoparticles and nanoclusters. J Biomed Nanotechnol 13:167-179. https://doi.org/10.1166/jbn.2017.2344

30. Golchin A, Rekabgardan M, Taheri RA, Nourani MR (2018) Promotion of cell-based therapy: special focus on the cooperation of mesenchymal stem cell therapy and gene therapy for clinical trial studies. In: Turksen K (ed) Advances in experimental medicine and biology. Springer, New York, pp 103-118

31. Golchin A, Shams F, Karami F (2020) Advancing mesenchymal stem cell therapy with CRISPR/Cas9 for clinical trial studies. Springer, New York

32. Basiri A, Mansouri F, Azari A, Ranjbarvan P et al (2021) Stem cell therapy potency in personalizing severe COVID-19 treatment. Stem Cell Rev Rep 17:193-213. https://doi.org/10.1007/ s12015-020-10110-w

33. Golchin A, Shams F, Kangari P et al (2019) Regenerative medicine: injectable cell-based therapeutics and approved products. Springer, Cham

34. Krishnan SR, Jaiswal R, Brown RD et al (2016) Multiple myeloma and persistence of drug resistance in the age of novel drugs (Review). Int J Oncol 49:33-50

35. Dey P, Rathod M, De A (2019) Targeting stem cells in the realm of drug-resistant breast cancer. Breast Cancer Targets Ther 11:115-135

36. Li Y, Ayala-Orozco C, Rauta PR, Krishnan S (2019) The application of nanotechnology in enhancing immunotherapy for cancer treatment: current effects and perspective. Nanoscale 11:17157-17178

37. Mckee SJ, Bergot AS, Leggatt GR (2015) Recent progress in vaccination against human papillomavirus-mediated cervical cancer. Rev Med Virol 25:54-71

38. Audran R, Peter K, Dannull J et al (2003) Encapsulation of peptides in biodegradable microspheres prolongs their MHC class-I 
presentation by dendritic cells and macrophages in vitro. Vaccine 21:1250-1255. https://doi.org/10.1016/S0264-410X(02)00521-2

39. Fridman WH, Zitvogel L, Sautès-Fridman C, Kroemer G (2017) The immune contexture in cancer prognosis and treatment. Nat Rev Clin Oncol 14:717-734

40. Zhang Y, Li N, Suh H, Irvine DJ (2018) Nanoparticle anchoring targets immune agonists to tumors enabling anti-cancer immunity without systemic toxicity. Nat Commun 9:1-15. https://doi. org/10.1038/s41467-017-02251-3

41. Gholami A, Hashemi SA, Yousefi K et al (2020) 3D nanostructures for tissue engineering, cancer therapy, and gene delivery. J Nanomater. https://doi.org/10.1155/2020/1852946

42. Ali OA, Emerich D, Dranoff G, Mooney DJ (2009) In situ regulation of DC subsets and $\mathrm{T}$ cells mediates tumor regression in mice. Sci Transl Med 1:8ra19. https://doi.org/10.1126/scitranslm ed.3000359

43. Gu L, Mooney DJ (2016) Biomaterials and emerging anticancer therapeutics: engineering the microenvironment. Nat Rev Cancer 16:56-66

44. Stephan SB, Taber AM, Jileaeva I et al (2015) Biopolymer implants enhance the efficacy of adoptive T-cell therapy. Nat Biotechnol 33:97-101. https://doi.org/10.1038/nbt.3104

45. Le Q-V, Yang G, Wu Y et al (2019) Nanomaterials for modulating innate immune cells in cancer immunotherapy. Asian $\mathrm{J}$ Pharm Sci 14:16-29. https://doi.org/10.1016/j.ajps.2018.07.003

46. Song M, Liu T, Shi C et al (2016) Bioconjugated manganese dioxide nanoparticles enhance chemotherapy response by priming tumor-associated macrophages toward M1-like phenotype and attenuating tumor hypoxia. ACS Nano 10:633-647. https:// doi.org/10.1021/acsnano.5b06779

47. Costa da Silva M, Breckwoldt MO, Vinchi F et al (2017) Iron induces anti-tumor activity in tumor-associated macrophages. Front Immunol. https://doi.org/10.3389/fimmu.2017.01479

48. Liu L, Yi H, He H et al (2017) Tumor associated macrophagetargeted microRNA delivery with dual-responsive polypeptide nanovectors for anti-cancer therapy. Biomaterials 134:166-179. https://doi.org/10.1016/j.biomaterials.2017.04.043

49. Meraz IM, Majidi M, Cao X et al (2018) TUSC2 Immunogene therapy synergizes with anti-PD-1 through enhanced proliferation and infiltration of natural killer cells in syngeneic Krasmutant mouse lung cancer models. Cancer Immunol Res 6:163177. https://doi.org/10.1158/2326-6066.Cir-17-0273

50. Jang E-S, Shin J-H, Ren G et al (2012) The manipulation of natural killer cells to target tumor sites using magnetic nanoparticles. Biomaterials 33:5584-5592. https://doi.org/10.1016/j.biomateria 1s.2012.04.041

51. Wesolowski R, Markowitz J, Carson WE (2013) Myeloid derived suppressor cells - a new therapeutic target in the treatment of cancer. J Immunother Cancer 1:10. https://doi.org/10.1186/ 2051-1426-1-10

52. Kong M, Tang J, Qiao Q et al (2017) Biodegradable hollow mesoporous silica nanoparticles for regulating tumor microenvironment and enhancing antitumor efficiency. Theranostics 7:3276

53. Mirza N, Fishman M, Fricke I et al (2006) All-trans-retinoic acid improves differentiation of myeloid cells and immune response in cancer patients. Cancer Res 66:9299-9307

54. Liu H, Mai J, Shen J et al (2018) A novel DNA aptamer for dual targeting of polymorphonuclear myeloid-derived suppressor cells and tumor cells. Theranostics 8:31

55. Borregaard N (2010) Neutrophils, from marrow to microbes. Immunity 33:657-670

56. Chu D, Zhao Q, Yu J et al (2016) Nanoparticle targeting of neutrophils for improved cancer immunotherapy. Adv Healthc Mater 5:1088-1093

57. Chu D, Dong X, Zhao Q et al (2017) Photosensitization priming of tumor microenvironments improves delivery of nanotherapeutics via neutrophil infiltration. Adv Mater 29:1701021

58. Li R, Fang F, Jiang M et al (2017) STAT3 and NF- $\kappa$ B are simultaneously suppressed in dendritic cells in lung cancer. Sci Rep 7:45395

59. Luo M, Wang H, Wang $Z$ et al (2017) A STING-activating nanovaccine for cancer immunotherapy. Nat Nanotechnol 12:648

60. Smits ELJM, Ponsaerts P, Berneman ZN, Van Tendeloo VFI (2008) The use of TLR7 and TLR8 ligands for the enhancement of cancer immunotherapy. Oncologist 13:859-875

61. Griesmann H, Drexel C, Milosevic N et al (2017) Pharmacological macrophage inhibition decreases metastasis formation in a genetic model of pancreatic cancer. Gut 66:1278-1285

62. Yuan H, Jiang W, Von Roemeling CA et al (2017) Multivalent bi-specific nanobioconjugate engager for targeted cancer immunotherapy. Nat Nanotechnol 12:763-769

63. Zhan X, Jia L, Niu Y et al (2014) Targeted depletion of tumourassociated macrophages by an alendronate-glucomannan conjugate for cancer immunotherapy. Biomaterials 35:10046-10057

64. Wang Y, Xu Z, Guo S et al (2013) Intravenous delivery of siRNA targeting CD47 effectively inhibits melanoma tumor growth and lung metastasis. Mol Ther 21:1919-1929

65. Wang Y, Lin Y-X, Qiao S-L et al (2017) Polymeric nanoparticles enable reversing macrophage in tumor microenvironment for immunotherapy. Biomaterials 112:3e163

66. Jambhrunkar M, Yang Y, Yu M et al (2020) Pristine large pore benzene-bridged mesoporous organosilica nanoparticles as an adjuvant and co-delivery platform for eliciting potent antitumor immunity. Mater Today Adv 6:100069. https://doi.org/10.1016/j. mtadv.2020.100069

67. Zanganeh S, Hutter G, Spitler R et al (2016) Iron oxide nanoparticles inhibit tumour growth by inducing pro-inflammatory macrophage polarization in tumour tissues. Nat Nanotechnol 11:986. https://doi.org/10.1038/nnano.2016.168

68. Rodell CB, Cuccarese MF, Garris CS, Li R, Ahmed MS et al (2018) TLR7/8-agonist-loaded nanoparticles promote the polarization of tumour-associated macrophages to enhance cancer immunotherapy. Nat Biomed Eng 2:578-588

69. Leuschner F, Dutta P, Gorbatov R et al (2011) Therapeutic siRNA silencing in inflammatory monocytes in mice. Nat Biotechnol 29:1005-1010. https://doi.org/10.1038/nbt.1989

70. Zhou S, Zhang T, Peng B et al (2017) Targeted delivery of epirubicin to tumor-associated macrophages by sialic acid-cholesterol conjugate modified liposomes with improved antitumor activity. Int J Pharm 523:203-216. https://doi.org/10.1016/j.ijpharm. 2017.03.034

71. Plebanek MP, Bhaumik D, Bryce PJ, Thaxton CS (2018) Scavenger receptor type B1 and lipoprotein nanoparticle inhibit myeloid-derived suppressor cells. Mol Cancer Ther 17:686-697. https://doi.org/10.1158/1535-7163.Mct-17-0981

72. Jeanbart L, Kourtis IC, van der Vlies AJ et al (2015) 6-Thioguanine-loaded polymeric micelles deplete myeloid-derived suppressor cells and enhance the efficacy of T cell immunotherapy in tumor-bearing mice. Cancer Immunol Immunother 64:10331046. https://doi.org/10.1007/s00262-015-1702-8

73. Kullberg M, Martinson H, Mann K, Anchordoquy TJ (2015) Complement $\mathrm{C} 3$ mediated targeting of liposomes to granulocytic myeloid derived suppressor cells. Nanomedicine 11:1355-1363. https://doi.org/10.1016/j.nano.2015.03.010

74. Sasso MS, Lollo G, Pitorre M et al (2016) Low dose gemcitabine-loaded lipid nanocapsules target monocytic myeloidderived suppressor cells and potentiate cancer immunotherapy. Biomaterials 96:47-62

75. Xiang J, Xu L, Gong H et al (2015) Antigen-loaded upconversion nanoparticles for dendritic cell stimulation, tracking, and 
vaccination in dendritic cell-based immunotherapy. ACS Nano 9:6401-6411. https://doi.org/10.1021/acsnano.5b02014

76. Chen Q, Xu L, Liang C et al (2016) Photothermal therapy with immune-adjuvant nanoparticles together with checkpoint blockade for effective cancer immunotherapy. Nat Commun 7:1-13

77. Han Q, Wang X, Jia X et al (2017) CpG loaded MoS2 nanosheets as multifunctional agents for photothermal enhanced cancer immunotherapy. Nanoscale 9:5927-5934. https://doi.org/10. 1039/c7nr01460k

78. Lee C, Jose L, Shim K et al (2019) Influenza mimetic proteinpolymer nanoparticles as antigen delivery vehicles to dendritic cells for cancer immunotherapy. Nanoscale 11:13878-13884. https://doi.org/10.1039/c9nr03510a

79. Zhang J, Shen L, Li X et al (2019) Nanoformulated codelivery of quercetin and alantolactone promotes an antitumor response through synergistic immunogenic cell death for microsatellitestable colorectal cancer. ACS Nano 13:12511-12524. https://doi. org/10.1021/acsnano.9b02875

80. Feng B, Zhou F, Hou B et al (2018) Binary cooperative prodrug nanoparticles improve immunotherapy by synergistically modulating immune tumor microenvironment. Adv Mater 30:e1803001. https://doi.org/10.1002/adma.201803001

81. Xue J, Zhao Z, Zhang L et al (2017) Neutrophil-mediated anticancer drug delivery for suppression of postoperative malignant glioma recurrence. Nat Nanotechnol 12:692. https://doi.org/10. 1038/nnano.2017.54

82. Tsai S, Santamaria P (2013) MHC class II polymorphisms, autoreactive T-cells, and autoimmunity. Front Immunol 4:321. https://doi.org/10.3389/fimmu.2013.00321

83. Stephan MT, Moon JJ, Um SH et al (2010) Therapeutic cell engineering with surface-conjugated synthetic nanoparticles. Nat Med 16:1035-1041. https://doi.org/10.1038/nm.2198

84. Kwong B, Gai SA, Elkhader J et al (2013) Localized immunotherapy via liposome-anchored Anti-CD137 + IL-2 prevents lethal toxicity and elicits local and systemic antitumor immunity. Cancer Res 73:1547-1558. https://doi.org/10.1158/0008-5472. Can-12-3343

85. Tsai S, Shameli A, Yamanouchi J et al (2010) Reversal of autoimmunity by boosting memory-like autoregulatory T cells. Immunity 32:568-580. https://doi.org/10.1016/j.immuni.2010.03.015

86. Xu M, Chen Y, Banerjee P et al (2017) Dendritic cells targeting and $\mathrm{pH}$-responsive multi-layered nanocomplexes for smart delivery of DNA vaccines. AAPS PharmSciTech 18:2618-2625. https://doi.org/10.1208/s12249-017-0741-1

87. Verbeke R, Lentacker I, Wayteck L et al (2017) Co-delivery of nucleoside-modified mRNA and TLR agonists for cancer immunotherapy: restoring the immunogenicity of immunosilent mRNA. J Control Release 266:287-300. https://doi.org/10. 1016/j.jconrel.2017.09.041

88. Pearson RM, Casey LM, Hughes KR et al (2017) Controlled delivery of single or multiple antigens in tolerogenic nanoparticles using peptide-polymer bioconjugates. Mol Ther 25:16551664. https://doi.org/10.1016/j.ymthe.2017.04.015

89. Liu L, Wang Y, Miao L et al (2018) Combination immunotherapy of MUC1 mRNA nano-vaccine and CTLA-4 blockade effectively inhibits growth of triple negative breast cancer. Mol Ther 26:4555. https://doi.org/10.1016/j.ymthe.2017.10.020

90. Huo M, Zhao Y, Satterlee AB et al (2017) Tumor-targeted delivery of sunitinib base enhances vaccine therapy for advanced melanoma by remodeling the tumor microenvironment. J Control Release 245:81-94. https://doi.org/10.1016/j.jconrel.2016.11.013

91. Ou W, Thapa RK, Jiang L et al (2018) Regulatory T cell-targeted hybrid nanoparticles combined with immuno-checkpoint blockage for cancer immunotherapy. J Control Release 281:84-96. https://doi.org/10.1016/j.jconrel.2018.05.018
92. Sacchetti C, Rapini N, Magrini A et al (2013) In vivo targeting of intratumor regulatory $\mathrm{T}$ cells using PEG-modified single-walled carbon nanotubes. Bioconjug Chem 24:852-858. https://doi.org/ 10.1021/bc400070q

93. Song W, Shen L, Wang Y et al (2018) Synergistic and low adverse effect cancer immunotherapy by immunogenic chemotherapy and locally expressed PD-L1 trap. Nat Commun 9:2237. https://doi.org/10.1038/s41467-018-04605-x

94. Duan X, Chan C, Han W et al (2019) Immunostimulatory nanomedicines synergize with checkpoint blockade immunotherapy to eradicate colorectal tumors. Nat Commun 10:1899. https://doi. org/10.1038/s41467-019-09221-X

95. Min Y, Roche KC, Tian S et al (2017) Antigen-capturing nanoparticles improve the abscopal effect and cancer immunotherapy. Nat Nanotechnol 12:877-882. https://doi.org/10.1038/nnano. 2017.113

96. Sung YC, Jin PR, Chu LA et al (2019) Delivery of nitric oxide with a nanocarrier promotes tumour vessel normalization and potentiates anti-cancer therapies. Nat Nanotechnol 14:11601169. https://doi.org/10.1038/s41565-019-0570-3

97. Mi Y, Smith CC, Yang F et al (2018) A dual immunotherapy nanoparticle improves T-cell activation and cancer immunotherapy. Adv Mater 30:e1706098. https://doi.org/10.1002/adma.20170 6098

98. Yang G, Xu L, Chao Y et al (2017) Hollow $\mathrm{MnO}_{2}$ as a tumormicroenvironment-responsive biodegradable nano-platform for combination therapy favoring antitumor immune responses. Nat Commun 8:902. https://doi.org/10.1038/s41467-017-01050-0

99. Chen Q, Wang C, Zhang X et al (2019) In situ sprayed bioresponsive immunotherapeutic gel for post-surgical cancer treatment. Nat Nanotechnol 14:89-97. https://doi.org/10.1038/ s41565-018-0319-4

100. Zhang C, Shi G, Zhang J et al (2017) Targeted antigen delivery to dendritic cell via functionalized alginate nanoparticles for cancer immunotherapy. J Control Release 256:170-181. https://doi.org/ 10.1016/j.jconrel.2017.04.020

101. Lu Y, Yang Y, Gu Z et al (2018) Glutathione-depletion mesoporous organosilica nanoparticles as a self-adjuvant and Co-delivery platform for enhanced cancer immunotherapy. Biomaterials 175:82-92. https://doi.org/10.1016/j.biomaterials. 2018.05.025

102. Zhang LX, Xie XX, Liu DQ et al (2018) Efficient co-delivery of neo-epitopes using dispersion-stable layered double hydroxide nanoparticles for enhanced melanoma immunotherapy. Biomaterials 174:54-66. https://doi.org/10.1016/j.biomaterials.2018. 05.015

103. Wang X, Li X, Ito A et al (2016) Stimulation of in vivo antitumor immunity with hollow mesoporous silica nanospheres. Angew Chemie 128:1931-1935. https://doi.org/10.1002/ange.20150 6179

104. Hu Q, Wu M, Fang C et al (2015) Engineering nanoparticlecoated bacteria as oral DNA vaccines for cancer immunotherapy. Nano Lett 15:2732-2739. https://doi.org/10.1021/acs.nanolett. 5 b00570

105. Stephan MT, Stephan SB, Bak P et al (2012) Synapse-directed delivery of immunomodulators using T-cell-conjugated nanoparticles. Biomaterials 33:5776-5787. https://doi.org/10.1016/j. biomaterials.2012.04.029

106. Kuai R, Yuan W, Son S et al (2018) Elimination of established tumors with nanodisc-based combination chemoimmunotherapy. Sci Adv 4:eaao1736. https://doi.org/10.1126/sciadv.aao1736

107. Rosalia RA, Cruz LJ, van Duikeren S et al (2015) CD40-targeted dendritic cell delivery of PLGA-nanoparticle vaccines induce potent anti-tumor responses. Biomaterials 40:88-97. https://doi. org/10.1016/j.biomaterials.2014.10.053 
108. Amanna IJ, Slifka MK (2011) Contributions of humoral and cellular immunity to vaccine-induced protection in humans. Virology 411:206-215. https://doi.org/10.1016/j.virol.2010.12.016

109. Sokolova V, Westendorf AM, Buer J et al (2015) The potential of nanoparticles for the immunization against viral infections. J Mater Chem B 3:4767-4779

110. Sarvaria A, Madrigal JA, Saudemont A (2017) B cell regulation in cancer and anti-tumor immunity. Cell Mol Immunol 14:662674. https://doi.org/10.1038/cmi.2017.35

111. Schmid D, Park CG, Hartl CA et al (2017) T cell-targeting nanoparticles focus delivery of immunotherapy to improve antitumor immunity. Nat Commun 8:1747

112. Temchura VV, Kozlova D, Sokolova V et al (2014) Targeting and activation of antigen-specific B-cells by calcium phosphate nanoparticles loaded with protein antigen. Biomaterials 35:6098-6105. https://doi.org/10.1016/j.biomaterials.2014.04. 010

113. Yan S, Gu W, Zhang B et al (2018) High adjuvant activity of layered double hydroxide nanoparticles and nanosheets in antitumour vaccine formulations. Dalt Trans 47:2956-2964

114. Martucci NM, Migliaccio N, Ruggiero I et al (2016) Nanoparticle-based strategy for personalized B-cell lymphoma therapy. Int J Nanomed 11:6089-6101. https://doi.org/10.2147/ijn.S118661

115. Watson DS, Endsley AN, Huang L (2012) Design considerations for liposomal vaccines: influence of formulation parameters on antibody and cell-mediated immune responses to liposome associated antigens. Vaccine 30:2256-2272. https://doi.org/10.1016/j. vaccine. 2012.01.070

116. Friede M, Muller S, Briand JP et al (1993) Induction of immune response against a short synthetic peptide antigen coupled to small neutral liposomes containing monophosphoryl lipid A. Mol Immunol 30:539-547. https://doi.org/10.1016/0161-5890(93) 90028-a
117. Stefanick JF, Ashley JD, Bilgicer B (2013) Enhanced cellular uptake of peptide-targeted nanoparticles through increased peptide hydrophilicity and optimized ethylene glycol peptide-linker length. ACS Nano 7:8115-8127. https://doi.org/10.1021/nn403 3954

118. Zilker C, Kozlova D, Sokolova V et al (2017) Nanoparticle-based B-cell targeting vaccines: tailoring of humoral immune responses by functionalization with different TLR-ligands. Nanomedicine 13:173-182. https://doi.org/10.1016/j.nano.2016.08.028

119. Kasturi SP, Skountzou I, Albrecht RA et al (2011) Programming the magnitude and persistence of antibody responses with innate immunity. Nature 470:543-547. https://doi.org/10.1038/ nature09737

120. Nikitin MP, Shipunova VO, Deyev SM, Nikitin PI (2014) Biocomputing based on particle disassembly. Nat Nanotechnol 99(9):716-722. https://doi.org/10.1038/nnano.2014.156

121. Yakovtseva MN, Betzer O, Lunin AV et al (2020) Magnetic and gold nanoparticles optimized for cancer treatment via cell hitchhiking. Proc_-Int Conf Laser Opt. https://doi.org/10.1109/ ICLO48556.2020.9285858

122. Fontana F, Liu D, Hirvonen J, Santos HA (2017) Delivery of therapeutics with nanoparticles: what's new in cancer immunotherapy? Wiley Interdiscip Rev Nanomed Nanobiotechnol 9:e1421

Publisher's Note Springer Nature remains neutral with regard to jurisdictional claims in published maps and institutional affiliations. 